\title{
LITERATURBERICHT
}

\section{Peacebuilding: am Scheideweg - oder in der Sackgasse?}

\author{
Sven Chojnacki / Anne Menzel
}

\begin{abstract}
Peacebuilding: At a Crossroads - or at a Dead End?
Abstract: We present a critical overview of the current debate on the use, scope, and effectiveness of peace building. Most notably, we analyze to what extent recent academic publications sufficiently reflect problematic aspects of current practices. The focus is on developments in the research on peace building concerning strategies, which have been and still are being implemented by political institutions and actors in the field: Local ownership and the coordination and integration of various peace building actors. We arrive at the conclusion that it is worthwhile practically as well as analytically necessary to give up concepts of social engeneering steering and alternatively develop ways to systematically cope with the lack of knowledge.
\end{abstract}

Keywords: Peace, Peacebuilding, Local Ownership, Coordination, Integration, Lack of Knowledge

Schlagwörter: Frieden, Peacebuilding, lokale Ownership, Koordination, Integration, Nichtwissen

\section{Einleitung}

Peacebuilding ist zugleich Norm und Praxis. Während die normative Dimension auf die moralisch begründete Notwendigkeit der kollektiven Bewältigung sicherheitspolitischer und sozio-ökonomischer Folgen von kriegerischer Gewalt verweist, richtet sich Peacebuilding als Praxis auf die Umsetzung konkreter Maßnahmen. Darunter fallen insbesondere der "Aufbau“ staatlicher Institutionen, Wirtschaftsförderung („Armutsreduktion“), die Durchführung von „freien“ Wahlen und auch die Entwaffnung, Demobilisierung und Reintegration von Kriegsbeteiligten unter besonderer Berücksichtigung von kollektiver „Versöhnungs“-Arbeit und strafrechtlicher Aufarbeitung der Vergangenheit. Angesichts der Permanenz von Interventions- „Notwendigkeiten“ in der internationalen Politik ist Peacebuilding heute kaum mehr von der weltpolitischen Bühne wegzudenken. Dabei wurde der so weitreichende Einsatz von Peacebuilding-Maßnahmen praktisch erst mit dem Ende des Ost-West-Konflikts vorstellbar. Zum einen ermöglichte die Auflösung der Blockkonfrontation die Konstruktion einer „westlich“ dominierten „internationalen Gemeinschaft“, in deren Namen fortan in die inneren Angelegenheiten kriegserschütterter Staaten eingegriffen werden konnte. In der 
„Agenda for Peace“ (1992), die auch als Peacebuilding-Gründungsdokument verstanden werden kann, brachte der damalige Generalsekretär der Vereinten Nationen Boutros Boutros-Ghali die neuen Opportunitätsstrukturen für kollektives Handeln auf den Punkt:

"Even as the issues between States north and south grow more acute, and call for attention at the highest levels of government, the improvement in relations between States east and west affords new possibilities, some already realized, to meet successfully threats to common security" (UN Secretary General 1992, Absatz 8).

Zum anderen lagen auch erst nach der weltpolitischen Zäsur von 1989/90 zumindest im Groben konsensfähige Ideen dazu vor, wie Nachkriegsländer durch externe Eingriffe zu gestalten sind: nämlich entlang der Leitmotive von Demokratie und Marktwirtschaft (vgl. Paris 2004, S. 15-16; Cockayne u. Malone 2005, S. 334-225; Baranyi 2008b, S. 9-10).

Dabei stand der Peacebuilding-Maßnahmen-Katalog keinesfalls von Anfang an unhinterfragt und unveränderlich fest. Vielmehr befinden sich PeacebuildingMaßnahmen seit Beginn der 1990er-Jahre in einem Prozess ständiger Revision, Differenzierung, Verfeinerung und Aufeinander-Abstimmung; und auch die Peacebuilding-Akteure - allen voran die Vereinten Nationen (UN) - befinden sich geradezu in permanenter Reform (vgl. etwa Lipson 2007; Jennings u. Kaspersen 2008). Die damit zeitweise verbundene Euphorie ist allerdings inzwischen einer deutlichen Ernüchterung gewichen. Auf der Basis einer mittlerweile zwei Jahrzehnte währenden Peacebuilding-Praxis und begleitender Forschung ist zunehmend deutlich geworden, dass Peacebuilding-Ergebnisse vielerorts trotz dauerhafter Bemühungen und immenser Ressourcenaufwendungen weit hinter den gesetzten Erwartungen zurückgeblieben sind - und zurückbleiben (vgl. etwa Mac Ginty 2006, S. 2-3; Paris u. Sisk 2009a, S. 11).

Aller Ernüchterung zum Trotz kommt Roland Paris (2010), dessen Arbeiten zu den einflussreichsten in der wissenschaftlichen Reflektion über Peacebuilding zählen, zu dem Schluss, dass Peacebuilding vor seinen Kritikerinnen und Kritikern gerettet werden müsse (Paris 2010, S. 339). Zwar begrüßt Paris, dass die von „irrationaler" Begeisterung geprägten Erwartungen der 1990er-Jahre mittlerweile passé seien, zugleich aber beunruhigt ihn offensichtlich der zunehmend kritische Unterton: So konstatiert er, dass die Ziele von Peacebuilding häufig falsch verstanden würden, durchaus konstatierbare Erfolge ignoriert und die moralische Komplexität der Entscheidung für oder gegen Peacebuilding von radikalen Kritikerinnen und Kritikern unzulässig simplifiziert werde (Paris 2010, S. 351-354). Mehr noch: In immer mehr wissenschaftlichen Publikationen werde Peacebuilding „fahrlässig“ mit den Wiederaufbau- und Stabilisierungsbemühungen im Zuge des „Kriegs gegen den Terror“ gleichgesetzt und/oder als neue Form von Kolonialismus porträtiert (Paris 2010, S. 348-350). Aufgrund solcher Pauschalisierungen und Vereinfachungen seien „hyper-kritische“ Haltungen sowohl ungerechtfertigt als auch im Hinblick auf ihre Konsequenzen - die Abschaffung von Peacebuilding - unüberlegt. Roland Paris sieht Peacebuilding daher an einem Scheideweg. Es müsse entweder ganz aufgegeben oder eben verbessert werden. 
Paris selbst plädiert für die zweite Option: „The challenge today is not to replace or move „beyond“ liberal peacebuilding, but to reform existing approaches within a broadly liberal framework “ (Paris 2010, S. 362). Mit einem „broadly liberal framework" verweist Paris dabei auf die - zumindest grobe - Ausrichtung von Peacebuilding auf einen „Aufbau“ demokratischer Staatlichkeit und wirtschaftliche Liberalisierung (vgl. Paris 2010, S. 361-363).

Ziel dieses Literaturberichts ist es im Folgenden nun nicht, die von Paris eingeforderte Reformdebatte zu rekonstruieren oder gar anzuführen. Stattdessen gehen wir noch einmal einen Schritt zurück und werfen einen kritischen Blick auf die angebotene Situationsdefinition: Ist die wissenschaftliche Reflektion über Peacebuilding tatsächlich an einem Scheideweg angelangt - oder steckt sie wohlmöglich bereits seit längerem in der Sackgasse? Im Gegensatz zum Scheideweg zeichnet sich die Sackgasse dadurch aus, dass der Weg vorwärts versperrt oder zumindest noch nicht frei geräumt ist. Weniger metaphorisch formuliert bedeutet dies, dass wir die ausgewählten wissenschaftlichen Veröffentlichungen daraufhin beleuchten werden, ob die derzeitige Forschung überhaupt geeignet ist, Probleme der Praxis angemessen zu reflektieren und Reformen zu empfehlen, die zu einer Verbesserung von Peacebuilding führen können.

Dazu klären wir im Folgenden zunächst, was in den Arbeiten des „akademischen Peacebuilding-Mainstream", auf die wir unsere Diskussion konzentrieren, überhaupt unter Peacebuilding verstanden wird. Darauf aufbauend zeigen wir, wie Verbesserungsbedarf im wissenschaftlichen Diskurs „reflektiert“ wird; die Anführungszeichen stehen dafür, dass die Beschaffenheit des Verbesserungsbedarfs in den meisten Studien tatsächlich eher suggeriert als reflektiert oder definiert wird. Insgesamt steht dabei aber offenbar vor allem die oberflächliche Qualität bzw. die mangelhafte Wirkungstiefe von Peacebuilding-Maßnahmen auf dem Prüfstand. Ein Umdenken erscheint vor allem dort nötig, wo die auf Peacebuilding zurückgeführte Qualität von „Frieden“ oberflächlich bzw. eher fragil bleibt (vgl. etwa Mac Ginty 2006, S. 2-3; Paris u. Sisk 2009a, S. 11; Richmond u. Franks 2007, S. 46). Demnach klafft eine Lücke zwischen der normativen und der praktischen Dimension von Peacebuilding - wobei „Frieden“, der ja im Zentrum der Norm von Peacebuilding steht, in aller Regel nicht näher definiert wird.

In einem weiteren Schritt diskutieren wir beispielhaft aktuelle Stellungnahmen der Peacebuilding-Forschung zu jenen Meta-Strategien oder auch Umsetzungsprinzipien, die in der politischen Praxis erklärtermaßen bereits für geeignet gehalten werden, die Effektivität von Peacebuilding zu erhöhen: die Herstellung lokaler Ownership sowie Koordination und Integration zwischen verschiedenen Peacebuilding-Akteuren bzw. zwischen den jeweils von ihnen umzusetzenden Peacebuilding-Maßnahmen. Beide Meta-Strategien bzw. Umsetzungsprinzipien werden in der akademischen Peacebuilding-Debatte ausgesprochen kritisch kommentiert. Dabei wird zumindest im Hinblick auf Koordination und Integration auch das grundlegende Problem thematisiert, dass es sich - wie aus unserer Sicht auch bei der Herstellung lokaler Ownership - um eine Maßnahme zur Wirkungssteuerung handelt, die außer Acht lässt, dass über die Wirkung von Peacebuilding schlicht kein Steuerungswissen zur Verfügung steht (vgl. Campbell 2008, S. 653; Paris 2009, S. 63). 
Der Umstand, dass aus diesem offen erklärten „Nichtwissen“ über die Wirkungen von Peacebuilding keine drastischen Konsequenzen gezogen werden, macht aus unserer Sicht die Sackgasse aus, in der die wissenschaftliche Forschung zu Peacebuilding heute steckt. Im Schlussteil legen wir daher nahe, dass es zum einen analytisch notwendig und zum anderen praktisch sinnvoll wäre, sozialtechnologische Steuerungsvorstellungen aufzugeben und stattdessen konkrete Möglichkeiten für die Auseinandersetzung mit „Nichtwissen“ im Peacebuilding zu erarbeiten. „Nichtwissen“ steht hier wiederum in Anführungszeichen, da in den letzten Jahren ohne Frage beeindruckende Wissensbestände über Peacebuilding erarbeitet worden sind. Nur stellen sie eben keine Grundlage dar, auf deren Basis die Wirkungen von Peacebuilding gesteuert werden könnten (vgl. ebenso Daxner et al. 2010, S. 12).

\section{Peacebuilding und der akademische Mainstream}

Angesichts der kaum überschaubaren Flut von Publikationen zu Peacebuilding, die sich heute in verschiedenen peer-reviewed Journals ${ }^{1}$, Monografien und Sammelbänden Bahn bricht, kann ein Literaturbericht nur selektive Einblicke vermitteln. Unsere Diskussion bezieht sich insofern auf eine Auswahl von größtenteils über den Zeitraum der letzten Jahre erschienenen Arbeiten, die PeacebuildingProbleme sowohl über eine „Mainstream-Perspektive“ erschließen als auch das Auseinanderklaffen von Norm und Praxis im Peacebuilding kritisch in den Blick nehmen.

Der akademische „Peacebuilding-Mainstream “ - so wie wir ihn hier verstehen - zeichnet sich zum einen dadurch aus, dass er zur praktischen Verbesserung von Peacebuilding beitragen will - und damit sowohl praxisnah als auch praxisrelevant ist. Die Praxisrelevanz der je erarbeiteten Ergebnisse stellt innerhalb des Peacebuilding-Mainstream nicht selten den zentralen Maßstab für „gute“ Forschung dar. „Gut“ ist demnach das, was praktisch nützlich ist bzw. was sich mit wohlbegründeter Aussicht auf praktische Verbesserungserfolge in Empfehlungsform an Politik und Praxis adressieren lässt. Der normative Druck, der dabei über dieses Gebot der Praxisrelevanz aufgebaut wird, lässt sich etwa daran ablesen, dass Arbeiten, die sich ihm in kritischer Absicht gänzlich entziehen, gerade auch für dieses „Versäumnis“ kritisiert werden (vgl. Paris 2010, S. 354); oder auch daran, dass Autorinnen und Autoren, die zum Zweck theoretischer und analytischer Grundlagenarbeit zumindest zeitweise Abstand von Praxisempfehlungen nehmen, immer wieder betonen, dass der Wert auch solcher Arbeiten nicht unterschätzt werden sollte (vgl. Daxner et al. 2010, S. 15).

Allerdings würden selbst die besten praxisbezogenen Empfehlungen nichts nützen, wenn nicht zugleich zumindest ein Minimum an Vertrauen in diejenigen gesetzt würde, die politische Entscheidungen über Peacebuilding treffen und für die

1 Auf Peacebuilding-Themen spezialisierte Journals sind etwa International Peacekeeping, Journal of Intervention and Statebuilding und das International Journal for Transitional Justice. Arbeiten zu Peacebuilding erscheinen zudem auch in thematisch auf Entwicklungs- oder Sicherheitspolitik, Friedens- und Konfliktforschung oder Internationale Beziehungen sowie in regionalwissenschaftlich ausgerichteten Journals. 
praktische Umsetzung von Peacebuilding verantwortlich sind. Entsprechend wird im akademischen Peacebuilding-Mainstream selten in Frage gestellt, dass die Peacebuilding-Akteure grundsätzlich mit „guten“ Intentionen tatsächlich darauf abzielen, in Nachkriegsgesellschaften Frieden zu ermöglichen; und zumindest implizit wird in der Regel davon ausgegangen, dass Weltbilder und politische Interessen der Peacebuilding-Akteure grundsätzlich mit den Zielen von Peacebuilding vereinbar sind.

Die Peacebuilding-Akteure werden dabei im akademischen Mainstream häufig simplifizierend unter der Bezeichnung „internationale Gemeinschaft“ zusammengefasst. Darunter fallen dann sowohl internationale NGOs als auch (halb-)staatliche Entwicklungszusammenarbeits-Agenturen (etwa DFID, USAID und GTZ bzw. mittlerweile GIZ), staatliche Regierungen und ihre Bürokratien (insbesondere die der USA, Kanadas, Großbritanniens, Frankreichs, Deutschlands, der Niederlande und der nordischen Länder) als auch internationale Organisationen (vor allem UN, OSCE, NATO, EU, Weltbank und IMF) (vgl. etwa Paris 2004, S. 2223; Fortna 2008, S. 8).

Wie einleitend bereits angedeutet wurde, wird „Frieden“, auf den durch praxisrelevante Forschung gemeinsam mit diesen Peacebuilding-Akteuren hingearbeitet werden soll, im Peacebuilding-Mainstream in aller Regel nicht genauer definiert. In jedem Fall aber soll er dauerhaft und selbsttragend („nachhaltig“) sein, so dass er auch dann weiter bestehen bleibt, wenn Peacebuilding-Akteure ein Nachkriegsland bereits wieder verlassen haben (vgl. etwa Doyle u. Sambanis 2006, S. 91-93). Zudem wird davon ausgegangen, dass der angestrebte „Frieden“ nur unter den „richtigen“ Bedingungen entstehen kann, die durch Peacebuilding demnach zumindest ein gutes Stück weit gefördert, wenn nicht sogar ermöglicht werden können. Unter „Peacebuilding“ selbst wird entsprechend die Gesamtheit der Bemühungen verstanden, die nach der formalen Beendigung von Kriegen unternommen werden, um diese Bedingungen herzustellen bzw., „[...] to identify and support structures that will tend to strengthen and solidify peace in order to avoid a relapse into conflict" (UN Secretary-General 1992, Paragraf 21). ${ }^{2} \mathrm{Zu}$ diesen Bemühungen gehören vor allem die finanzielle, logistische und technische Unterstützung bei a) der Entwaffnung, Demobilisierung und Reintegration von Kombattanten (Disarmament, Demobilisation, Reintegration; DDR) ${ }^{3}$, b) Sicher-

2 Im Vergleich dazu werden in Arbeiten zu „Conflict Transformation“, die sich tendenziell eher mit dem Entstehen als dem Gefördert-Werden von Frieden beschäftigen, mit „Peacebuilding “ häufig gerade nicht in erster Linie extern verschriebene Maßnahmen bezeichnet. Stattdessen meint „Peacebuilding“ in ihnen häufig „sozialen Wandel hin zu Frieden“ - unabhängig davon, ob dieser Wandel durch extern verschriebene Maßnahmen bewirkt wurde oder nicht (vgl. etwa Lederach 2005, S. 34-40; Dayton u. Kriesberg 2009, S. 5; Dayton 2009, S. 61-62).

3 Einen recht guten Überblick über den aktuellen Forschungsstand zu DDR gibt der von Muggah (2009) herausgegebene Sammelband. Der Band enthält allerdings keinen Beitrag zu Fragen dazu, inwieweit Mädchen und Frauen in bewaffneten Gruppen und Armeen Zugang zu DDR-Programmen erhalten bzw. de facto bislang beim Zugang zu diversen DDR-Programmen erheblich benachteiligt waren. Vgl. hierzu McKay u. Mazurana (2004) und Coulter et al. (2008). 
heitssektor-, Justiz- und „local governance“-Reformen ${ }^{4}$, c) der Abhaltung von Wahlen und gegebenenfalls auch bei der Ausarbeitung von Verfassungen ${ }^{5}$, d) der Entwicklung von Strategien zur Armutsreduzierung (Poverty Reduction Strategy Papers; PRSP $)^{6}$ sowie e) der Entwicklung und Umsetzung von Strategien für einen friedlichen und friedenfördernden Umgang mit der gewaltsamen Vergangenheit (Transitional Justice). ${ }^{7}$ Diese Bemühungen müssen - wie Paris und Sisk (2009a) hervorheben - nicht notwendigerweise auf „western-style democracy or ,neoliberal" market ideologies" hinauslaufen; allerdings sind dies dennoch diejenigen Leitbilder, auf die Peacebuilding-Bemühungen de facto in aller Regel ausgerichtet sind (Paris u. Sisk 2009a, S. 15; vgl. auch Paris 2010, S. 360-361).

Die Bezeichnung „Peacebuilding-Mainstream“ wird hier folglich als analytische Klassifizierung verwendet, für die drei Kriterien angelegt werden: zum einen die Orientierung am Gebot der Praxisrelevanz; zweitens hinsichtlich der Prämisse „guter" Intentionen aufseiten der Peacebuilding-Akteure - verbunden mit der Annahme einer weitgehenden Vereinbarkeit ihrer Weltsichten und Interessen mit den Zielen von Peacebuilding sowie der Anerkennung durch die Adressaten bestimmter Peacebuilding-Maßnahmen, ohne die es wenig Sinn machen würde, praxisrelevante Empfehlungen an sie zu adressieren; und drittens die Zielvorstellung eines „Friedens“, der dauerhaft und selbsttragend sein und unter den „richtigen“ Bedingungen entstehen soll, die zumindest ein Stück weit durch Peacebuilding geschaffen werden können. Insofern, als eindeutig ein Großteil der wissenschaftlichen Arbeiten zu Peacebuilding diese Kriterien zumindest grob erfüllt, erscheint die Bezeichnung als „Mainstream“ angemessen.

Deutlich außerhalb des Peacebuilding-Mainstreams stehen - an diesen Kriterien bemessen - etwa die Arbeiten von Mark Duffield (2002, 2005, 2008). Ausgangspunkt von Duffields Analysen ist eine alternative Situationsdefinition. Ihr zufolge sollten Interventionen, die dem Namen nach zum Zweck von „Hilfe“ oder „Entwicklung “ oder auch „Wiederaufbau“ durchgeführt werden, besser als Bemühungen verstanden werden, um die Kontrolle und Einhegung jener Gefahren zu gewährleisten, die von unterentwickelten Konfliktregionen für das „versicherte Leben“ (, insured life“) der Bevölkerungen des „Westens“ ausgehen; darunter fallen u. a. Flüchtlingsströme, organisierte Kriminalität, Terrorismus und ansteckende

4 Diese drei Arten von Reformen weisen enge konzeptionelle Verbindungen zueinander auf und werden hier deshalb in einem Zuge genannt, vgl. für einen Überblick den von Call (2007a) herausgegebenen Sammelband. Als eine der aufwendigsten Sicherheitssektorreformen (SSR) gilt diejenige in Sierra Leone (Bendix u. Stanley 2008; Albrecht u. Jackson 2009).

5 Einen brauchbaren Überblick über den Forschungsstand zu unterschiedlichen Aspekten der Demokratisierung von Nachkriegsländern gibt der von Jarstad und Sisk (2008) herausgegebene Sammelband; vgl. außerdem den von De Zeeuw (2007) herausgegebenen Sammelband für Beiträge zu mehr oder weniger erfolgreichen Transformationen von Rebellenbewegungen zu politischen Parteien.

6 PRSPs werden sowohl in Nachkriegsländern als auch in „nur“ armen Ländern mit Unterstützung des Internationalen Währungsfonds (IMF) und der Weltbank erarbeitet. Die bislang erarbeiteten PRSPs sind im Internet von „A“ wie Afghanistan bis „Z“ wie Zambia abrufbar (vgl. http://www. imf.org/external/np/prsp/prsp.asp; zugegriffen 03.11.2010).

7 Einen Überblick über den aktuellen „state of the field“ gibt der gleichnamige Artikel von Bell (2009). Für aktuelle empirische Studien zu Transitional-Justice-,Instrumenten“, vor allem Wahrheitskommissionen und Strafverfolgung vgl. die Homepage des International Center for Transitional Justice (http://www.ictj.org/en/news/pubs/index.html; zugegriffen 03.11.2010). 
Krankheiten (Duffield 2008, S. 149). Duffield argumentiert, dass die HerrschaftsArrangements, die über solche vordergründig „gut" gemeinten Interventionen in den krisengeschüttelten „borderlands“ abseits der „westlichen“ Welt installiert würden, sich tatsächlich kaum von kolonialen Formen indirekter Herrschaft unterscheiden ließen. Es gehe in ihnen im Wesentlichen darum, in unterentwickelten Konfliktregionen Akteure zu stärken, die aus „westlicher" Perspektive willig und geeignet erscheinen, um mit ihnen ein Bollwerk gegen unkontrollierbare Elemente in den „borderlands“ zu errichten (Duffield 2002, S. 1052). In der Sprache kolonialzeitlicher indirekter Herrschaft ausgedrückt, handele es sich dabei schlicht um „the art of getting savages to fight barbarians" - also um die Kunst, die berechenbaren „Wilden“ einzusetzen, um die Unberechenbaren in Schach zu halten (Duffield 2005, S. 145). Die Tatsache der ungleichen Verteilungen von Lebenschancen zwischen den Bevölkerungen im „Westen“ und denen in den „borderlands“ werde dabei nicht nur weiterhin als gegeben hingenommen. Die Interventionen des „Westens" seien aufgrund der ihnen in Problemdefinitionen und Lösungskonzepten stets zugrundeliegenden "security mentality“ vielmehr darauf ausgerichtet, diese Ungleichheit aufrecht zu erhalten: indem sie die permanenten Krisen in unterentwickelten Konfliktregionen einhegen würden, ohne jedoch zu ihre Überwindung beitragen zu können oder zu wollen (Duffield 2005, S. 157).

Der zentrale Unterschied zum Peacebuilding-Mainstream besteht folglich darin, dass Duffield nicht als gegeben voraussetzt, dass Interventionen, die von ,westlichen "Sicherheitsinteressen geprägt sind, das Leben der Menschen in Nachkriegsländern und unterentwickelten Regionen verbessern können. Entsprechend sieht er auch keinen Anlass dazu, Handlungsempfehlungen zur Optimierung dieser Interventionen zu formulieren. Seine Arbeiten zielen vielmehr darauf ab, ein Bewusstsein für die interessengeladenen (bzw. in einem Foucaultschen Sinne vermachteten) Konzepte und Denkmuster zu schaffen, mit denen sowohl in der Peacebuilding-Praxis als auch in der Mainstream-Forschung meist ganz selbstverständlich gearbeitet wird (vgl. Duffield 2005, S. 145-146). Im akademischen Peacebuilding-Mainstream wird zwar durchweg anerkannt, dass die Sicherheitsinteressen „westlicher“ Staaten in Peacebuilding einfließen. Zugleich wird allerdings davon ausgegangen, dass diese Interessen mit „guten“ Intentionen grundsätzlich vereinbar sind. Denn schließlich sei Peacebuilding in jedem Fall darauf ausgerichtet, zum einen unabhängige - und nicht etwa unter ausbeuterischer Kolonialherrschaft gehaltene - und zum anderen friedliche Staaten hervorzubringen (vgl. Paris 2010, S. 349-350).

Ebenfalls abseits des Mainstreams stehen demgegenüber Arbeiten, in denen empfohlen wird, die Souveränität von Nachkriegsländern bzw. von „schwachen“ oder „kollabierten“ Staaten tatsächlich auf Dauer einzuschränken. In ihnen wird von der Situationsdefinition ausgegangen, dass bisherige Peacebuilding-Bemühungen, in denen die Einschränkung staatlicher Souveränität von vornherein nur auf begrenzte Zeiträume angelegt ist, überwiegend keine verlässlichen Erfolge liefern konnten und es deshalb an der Zeit sei, radikalere Policy-Optionen in Erwägung zu ziehen (vgl. Krasner 2004; Caplan 2007):

"De facto trusteeships and especially shared sovereignty arrangements that were legitimated by international legal sovereignty and sustained either by external enforce- 
ment or domestic equilibria, would offer political leaders a better chance of bringing peace and prosperity to the populations of badly governed states and reducing the threats which such polities present to the wider international community" (Krasner 2004, S. 41).

Anders als bei Duffield bleibt die Prämisse ,guter“ Intentionen in dieser Position zwar unangetastet, aber im Vergleich zum Mainstream fehlt ihr die hartnäckige Ausrichtung auf das Ziel, in den je betroffenen Gesellschaften tiefgreifende Veränderungen zu bewirken, die ein andauerndes Engagement externer Akteure in absehbarer Zeit obsolet machen würden.

Allerdings hat sich auch im Peacebuilding-Mainstream mittlerweile nachdrücklich die Überzeugung durchgesetzt, dass solche tiefgreifenden Veränderungen offenbar nicht von heute auf morgen und nicht insgesamt auf einmal erreicht werden können - und womöglich auch nicht alle auf einmal erreicht werden müssen. So wird beispielweise recht einhellig davon ausgegangen, dass insbesondere die Einführung von demokratischem Wettbewerb erst unter Bedingungen funktionierender Staatlichkeit, vor allem eines verlässlichen Gewaltmonopols, friedensfördernd wirkt. Unter Abwesenheit dieser Bedingungen würde die Gefahr eines Rückfalls in kriegerische Gewalt durch das Abhalten von Wahlen hingegen eher erhöht, indem über Wahlkämpfe bestehende Konflikte und Polarisierungen geschürt würden, ohne dass eine gewaltlose Austragung sichergestellt werden könnte (vgl. etwa Paris 2004, S. 151-152; Jarstad 2008b, S. 28-29; Reilly 2008, S. 161162). Als Alternative zu einem schnellen Abhalten von Wahlen plädiert Michael Barnett (2006) deshalb für ein „republican peacebuilding“. Dieses soll sich in seiner praktischen Umsetzung dadurch auszeichnen, dass Nachkriegsländern die „Schocktherapie“ (Barnett 2006, S. 90) schneller Demokratisierungsbemühungen unmittelbar nach Kriegsende vorerst erspart bleibe. Stattdessen, so argumentiert Barnett, müsse der Fokus gerade unmittelbar nach Kriegsende zunächst auf den „Aufbau“ legitimer, repräsentativer und vor allem stabiler staatlicher Institutionen gelegt werden. Peacebuilding müsse insofern zunächst vor allem Statebuilding sein (Barnett 2006, S. 89). Als ein empirisches Beispiel für die praktische Umsetzung eines republican peacebuilding, über das es gelungen sei, auch ohne potenziell destabilisierende Wahlen ein Mindestmaß an Legitimität für im „Aufbau“ befindliche staatliche Institutionen herzustellen, nennt Barnett die Einsetzung der Emergency Loya Jirga in Afghanistan. Über diesen nach Klanzugehörigkeitskriterien grob repräsentativ gestalteten Ältestenrat wurde in 2002 eine afghanische Übergangsregierung gewählt (Barnett 2006, S. 102-103). ${ }^{8}$

Barnetts Plädoyer für ein Statebuilding-fokussiertes Peacebuilding stellt dabei allerdings keine Abweichung vom Mainstream dar - im Gegenteil: Die meisten Mainstream-Autorinnen und -Autoren würden seine Ausführungen wohl ohne Zögern unterschreiben (vgl. etwa Manning 2004, S. 62-63; Paris 2004, S. 188189, 2010, S. 355; Jarstad 2008b, S. 28-29; Reilly 2008, S. 161-162). Dies gilt

8 In einer späteren Arbeit nimmt Barnett seinen Enthusiasmus für die Emergency Loya Jirga allerdings wieder ein gutes Stück weit zurück: "The results of the Loya Jirga were mixed: while major representatives of almost all Afghan groups agreed on the composition of the Transitional Authority, the actual negotiations were far from fair and transparent" (Barnett u. Zürcher 2009, S. 43). 
umso mehr, als auch Barnett eine demokratische Zukunft absolut nicht ausschließt: Er argumentiert vielmehr, dass Nachkriegsländer, in denen der Fokus zunächst darauf gelegt werde, legitime, repräsentative und vor allem stabile staatliche Institutionen „,aufzubauen“, bessere Aussichten darauf hätten, sich demokratisch zu entwickeln als solche, in denen schnelle Wahlen abgehalten würden, selbst wenn der Bevölkerung noch kein gewaltloser Ablauf garantiert werden könnte (vgl. Barnett 2006, S. 90).

Ebenfalls im Rahmen des Mainstreams bewegen sich auch jene Überlegungen innerhalb der wissenschaftlichen Debatte, die indigene/traditionelle PeacebuildingMethoden in die Bemühungen externer Akteure zu integrieren versuchen (vgl. auch Paris 2010, S. 358-359). Befürworter solcher Ansätze, wie etwa Roger Mac Ginty (2008), schlagen keinesfalls vor, die Peacebuilding-Bemühungen der „internationalen Gemeinschaft" abzubrechen. Vielmehr wird dafür plädiert, das Potenzial indigener/traditioneller Ansätze zur Konfliktbearbeitung und Friedensermöglichung zu erkennen und zur Effektivitätssteigerung von Peacebuilding zu nutzen. Unter „indigenen“ Methoden versteht Mac Ginty dabei „lokal inspirierte“ Ansätze, während "traditionelle" Methoden - die aber durchaus auch zugleich indigen sein können - sich in erster Linie dadurch auszeichnen sollen, dass sie lokal bereits lange Zeit in Gebrauch sind, ohne dabei jedoch notwendigerweise lokalen Ursprungs sein zu müssen (Mac Ginty 2008, S. 149-150): Eine Integration indigener/ traditioneller Methoden könne zum einen dazu beitragen, das "top-down bias“ konventioneller Peacebuilding-Maßnahmen zu überwinden - also den Umstand, dass die meisten Menschen in Nachkriegsländern Peacebuilding-Maßnahmen in der Tat eher selten direkt erleben, geschweige denn aktiv an ihnen beteiligt sind. Für indigene/traditionelle Methoden sei hingegen häufig das Gegenteil der Fall: „Many customary dispute resolution techniques are participatory and operate at precisely the community and local levels that top-down peace-making may fail to reach “ (Mac Ginty 2008, S. 158). Die relativen Vorteile indigener/traditioneller Methoden bestünden zudem darin, dass sie von der je betroffenen Bevölkerung vielfach eher als legitim angesehen würden als von externen Akteuren eingeführte Verfahren (vgl. Mac Ginty 2008, S. 155). Zugleich warnt Mac Ginty allerdings davor, indigene/traditionelle Methoden im Umkehrschluss zu romantisieren und per se für „westlichem“ Peacebuilding überlegen zu halten. Vielmehr sei stets davon auszugehen, dass auch indigene/traditionelle Methoden normativ problematisch und im Hinblick auf die Ziele von Peacebuilding dysfunktional sein können. Als empirisches Beispiel für eine normativ problematische und dysfunktionale Methode, die dennoch in Peacebuilding integriert wurde, führt Mac Ginty die GacacaTribunale in Ruanda an, in denen Opfer und Täter des Genozids dazu angehalten wurden, auf lokaler Ebene „restorative Gerechtigkeit“ miteinander auszuhandeln (Mac Ginty 2008, S. 149; vgl. auch Buckley-Zistel 2005). Für ein erfolgreiches Beispiel hingegen verweist auch Mac Ginty auf die Emergency Loya Jirga 2002 sowie auf die 2003 an sie anschließende, ebenfalls Peacebuilding-integrierte Loya Jirga, in der über eine afghanische Verfassung beraten wurde: „Although the scene of disputes and high drama, both Loya Jirgas succeeded in facilitating consensus decision-making." Allerdings weist Mac Ginty auch darauf hin, dass das Konzept indigener/traditioneller Methoden gerade am Beispiel der Loya Jirgas an seine 
Grenzen getrieben wurde. Da die Abhaltung der letzten „regulären“ (nicht Peacebuilding-integrierten) Loya Jirga 2002 bereits 40 Jahre zurück lag, hatten die meisten Menschen in Afghanistan zu diesem Zeitpunkt tatsächlich überhaupt keine eigenen Erfahrungen mit bzw. Erinnerungen an diese indigene/traditionelle Methode (Mac Ginty 2008, S. 156).

Dafür, dass Peacebuilding laut Paris (2010) deutlich von den Wiederaufbauund Stabilisierungsbemühungen im Zuge des „Kriegs gegen den Terror“" zu unterscheiden sei, finden sich in der Mainstream-Debatte überhaupt erstaunlich viele Bezugnahmen auf die militärischen und zivilen Komponenten der Interventionen in Afghanistan und, allerdings weniger häufig, auf den Krieg und die anschließenden Wiederaufbau- und Stabilisierungsbemühungen im Irak (vgl. etwa Call 2007b, S. 3). Ein genauerer Blick auf das bei Paris formulierte Differenzierungsargument zeigt jedoch, dass der Unterschied zwischen Peacebuilding und Wiederaufbau sowie Stabilisierung im Rahmen des „Kriegs gegen den Terror“ ohnehin gar nicht so sehr in den jeweils eingesetzten Maßnahmen bestehen soll, sondern in den Arbeitsbedingungen für die Umsetzung dieser Maßnahmen. Da externe Akteure in „PostEroberungskontexten" wie im Irak tendenziell als Besatzer und ihre Stabilisierungsmaßnahmen als aufgezwungen wahrgenommen würden, sei die Umsetzung extern verschriebener Maßnahmen hier sehr viel schwieriger als dies im Vergleich in den meisten Peacebuilding-Kontexten der Fall sei (Paris 2010, S. 348). Ob sich die Arbeitsbedingungen für Peacebuilding an den Schauplätzen des „Kriegs gegen den Terror" aber tatsächlich so grundlegend von denen in anderen Einsatzgebieten unterscheiden lassen, ist unserer Kenntnis nach noch gar nicht empirisch-vergleichend untersucht worden. Grundsätzlich wäre beispielsweise auch denkbar, dass jene Peacebuilding-Bemühungen vor ganz ähnlichen Problemen stehen, die zwar abseits der unmittelbaren Schauplätze des „Kriegs gegen den Terror“ umgesetzt werden, aber ähnlich wie vielerorts in Afghanistan oder auch im Irak de facto nicht mit „Nachkriegsproblemen“, sondern mit andauernder kriegerischer Gewalt und der Nicht-Anerkennung externer „Friedensakteure“ konfrontiert sind.

Auch der von Stephen Baranyi (2008a) herausgegebenen Sammelband „The Paradoxes of Peacebuilding Post-9/11 “ enthält trotz des vielversprechenden Titels keine solchen empirisch-vergleichenden Untersuchungen. Allerdings argumentiert Baranyi (2008b) einleitend, der „Krieg gegen den Terror“ habe tatsächlich auch die inhaltliche Ausrichtung von Peacebuilding-Maßnahmen geprägt. Gerade die Vorstellung einer „Responsibility to Protect“ habe nach dem 11. September 2001 keine Chance mehr gehabt, das Denken über und die Praxis von Peacebuilding zu bestimmen. ${ }^{9}$ Beides werde stattdessen von „westlich“ dominierten Sicherheitsdis-

9 Die „Responsibility to Protect“ ist der Entwurf eines völkerrechtlichen Prinzips zum Schutz von Zivilbevölkerungen, welches externe Interventionen in souveräne Staaten explizit erlaubt, sollten Staaten ihre eigenen Zivilbevölkerungen nicht schützen (können) oder sogar selbst Gewalt gegen sie richten. Mit Blick auf Peacebuilding ist insbesondere von Bedeutung, dass diese Verantwortung nicht nur im unmittelbaren Gefahrenmoment greifen soll, sondern auch die Prävention von Gewalt gegen Zivilbevölkerungen und den Wiederaufbau nach Gewalt gegen Zivilbevölkerungen bzw. nach externen Interventionen beinhaltet (ICISS 2001, S. 17). Die Responsibility to Protect wurde durch die von der kanadischen Regierung eingesetzte International Commission on Intervention and State Sovereignty (ICISS) umfangreich ausgearbeitet und in einem Report veröffentlicht, der kurz nach den Anschlägen vom elften September 2001 erschienen ist. 
kursen bestimmt (Baranyi 2008b, S. 11-12). Dieser Fokus auf „westliche“ Sicherheitsinteressen habe allerdings, so führt Baranyi weiter aus, den Themen „fragile Staatlichkeit" und Statebuilding ein erhöhtes Ausmaß an Aufmerksamkeit eingebracht, welches sie im Rahmen einer Selbstverpflichtung der ,internationalen Gemeinschaft" zum grenzüberschreitenden Schutz von Zivilbevölkerungen bestimmt nicht erhalten hätten: „The events of 9/11 have certainly pushed issues of state fragility up the international agenda in a way that proponents of R2P [Responsibility to Protect, Anm. SC/AM] can only envy“ (Baranyi 2008b, S. 16). Die Diagnose einer Sicherheits-,,Schlagseite“ von Peacebuilding-Maßnahmen wird jedoch weder von Baranyi (2008a) noch in den übrigen Beiträgen des Sammelbands zum Anlass genommen, wie Duffield $(2002,2005,2008)$ eine grundlegend Peacebuilding-skeptische Position zu beziehen. Sie bewegen sich vielmehr fest auf dem Boden des akademischen Peacebuilding-Mainstreams. Die Autorinnen und Autoren adressieren ihre Verbesserungsvorschläge und Visionen weiterhin an die ,internationale Gemeinschaft" und fordern dabei eher mehr als weniger Peacebuilding (vgl. Baranyi u. Powell 2008, S. 315). ${ }^{10}$

\section{Verbesserungsbedarf unter der Oberfläche}

In den Publikationen des akademischen Peacebuilding-Mainstreams beginnt die Feststellung von Verbesserungsbedarf nicht selten mit einer Erfolgsmeldung: Es gilt inzwischen als gesicherte Erkenntnis, dass Friedensmissionen, die während eines andauernden Krieges als militärische und politische Interventionen ansetzen und nach formalen Kriegsbeendigungen als Peacebuilding fortgeführt werden, zumindest insofern einen Unterschied machen, als sie das Risiko eines Rückfalls in kriegerische Gewalt für Nachkriegsländer statistisch deutlich senken (vgl. Call u. Cousens 2008, S. 8; Fortna u. Howard 2008, S. 284). Quantitativ-vergleichend untersucht worden ist die Frage nach dem Erfolg von Friedensmissionen etwa von Doyle und Sambanis (2006) und Fortna (2008). Beide Arbeiten kommen zu dem Schluss, dass Friedensmissionen statistisch signifikant mit dauerhaftem NichtKrieg in Nachkriegsländern korrelieren. ${ }^{11}$ Doyle und Sambanis finden sogar Hinweise darauf, dass gerade UN-Friedensmissionen dann erfolgreich sind, wenn sie zusätzlich noch mit einem Mehr an politischen Partizipationsmöglichkeiten in Nachkriegsländern einhergehen (Doyle u. Sambanis 2006, S. 88). Auch dieses Ergebnis wird durch Fortnas Untersuchung tendenziell bestätigt; bei ihr ist diese spezielle Korrelation allerdings statistisch nicht signifikant (Fortna 2008, S. 117, 174).

Trotz der somit in quantitativ-vergleichender Hinsicht eher positiven Bilanz erscheint die Qualität der sozialen Verhältnisse, die dort entstehen, wo Peacebuilding-Maßnahmen umgesetzt werden, immer noch weit von einem Ergebnis ent-

10 Anders als der Titel des Sammelbandes suggeriert, wird in den darin zusammengeführten Fallstudien nicht explizit untersucht, wie sich Peacebuilding unter Vorzeichen des „Kriegs gegen den Terror" verändert hat. Am ehesten scheint ihr gemeinsames Thema darin zu bestehen, dass auf die friedensfördernden Potenziale lokaler, ,zivilgesellschaftlicher' Akteure hingewiesen wird.

11 Die militärischen Interventionen in Afghanistan und in den Irak im Rahmen des „Kriegs gegen den Terror" werden in beiden Arbeiten nicht als Friedensmissionen gezählt. 
fernt, das im akademischen Peacebuilding-Mainstream als echter und überzeugender Erfolg gewertet würde. Roger Mac Ginty (2006, S. 2-3) beschreibt das Problem folgendermaßen: „[T]he peace that prevails is often prefixed with terms attesting to its compromised quality: 'brittle', 'fragile', 'turbulent', 'armed', 'nervous' and so on. [...] Rather than peace, many post-accord societies experience a 'no war, no peace' situation. " Und Paris und Sisk (2009a) beklagen, dass selbst für Situationen, die wie Peacebuilding-Erfolge wirken, kaum zu beurteilen sei, inwieweit der Erfolg tatsächlich „echt“ ist, worauf er beruht und ob er von Dauer sein kann; die Erfahrungen der Vergangenheit böten hier zudem keine eindeutigen Anlässe zu Optimismus (vgl. Paris u. Sisk 2009a, S. 11).

Ohne dass dies im akademischen Peacebuilding-Mainstream tatsächlich im Detail ausgeführt und definiert würde, wird Verbesserungsbedarf vor allem hinsichtlich einer wahrgenommenen Oberflächlichkeit und fehlenden Tiefenwirkung von Peacebuilding-Maßnahmen gesehen. Die Ergebnisse entsprechen vielfach offenbar in Art und Inhalt nicht den tiefgreifenden Veränderungen, die Peacebuilding dem Verständnis des akademischen Mainstreams nach - eigentlich hervorbringen sollte. Dazu, was diese Oberflächlichkeit konkret ausmacht, sind im akademischen Mainstream allerdings keine expliziten Überlegungen zu finden. Wohl aber ist vielfach empirisch untersucht und vor Kurzem erst modellhaft dargestellt worden, wie Oberflächlichkeit zustande kommt.

\subsection{Oberflächlichkeit als Ergebnis von externem Versagen}

Das Zustandekommen der Oberflächlichkeit von Peacebuilding-Ergebnissen ist bislang vor allem in jenen Einzelfallstudien beschrieben worden, in denen die Umsetzung einzelner Peacebuilding-Maßnahmen oder auch ganzer Peacebuilding-Projekte (Maßnahmen-Bündel) in Nachkriegsländern in den Blick genommen wird. In solchen Untersuchungen werden die Wirkungsannahmen hinter einzelnen Peacebuilding-Maßnahmen bzw. hinter ganzen Peacebuilding-Projekten mit den Bedingungen im jeweiligen Nachkriegsland und mit der praktischen Umsetzung von Peacebuilding abgeglichen. Über diesen Abgleich wird dann die Frage beantwortet, ob berechtigter Grund zu der Annahme besteht, dass Peacebuilding in intendierter Weise wirken könnte bzw. hätte wirken können. Hier nur einige Beispiele:

Carrie Manning (2004) etwa kommt in ihrer Untersuchung der Abhaltung von Wahlen und der Ausgestaltung demokratischer, politischer Institutionen (,institutional engeneering") in Bosnien und Herzegowina zu dem Schluss, dass nicht nur die Wirkungsannahmen und Ziele hinter diesen Maßnahmen unrealistisch waren (Manning 2004, S. 67-68, 83-84). Sie argumentiert, zusätzlich sei auch ihre Umsetzung planlos, unkoordiniert und von politischen Interessen einzelner Peacebuilding-Akteure geprägt gewesen. Insbesondere die Clinton-Administration hatte unmittelbar nach dem Ende des Krieges 1996 auf ein baldiges Abhalten von Wahlen in Bosnien und Herzegowina gedrängt; und dies offenbar vor allem auch deshalb, weil einer zunehmend interventions-skeptischen US-Wählerschaft vor den ebenfalls in 1996 anstehenden US-Präsidentschaftswahlen eine glaubhafte „exitstrategy" für Bosnien und Herzegowina präsentiert werden sollte. Dabei sei zu diesem Zeitpunkt bereits deutlich absehbar gewesen, dass Wahlen so kurz nach 
Kriegsende und unter Bedingungen anhaltender Unsicherheit in jedem Fall auf eine Bestätigung der Macht ethno-nationalistischer Parteien hinauslaufen würden - was dann auch eintrat. Wählerinnen und Wähler trauten dem „Frieden“ nicht und hielten fest zu „ihren“ jeweiligen ethno-nationalistischen Parteien (Manning 2004, S. 67-68). Die eigentlich intendierte tiefgreifende Veränderung, nämlich die Überwindung von verhärteten, ethno-nationalistischen Konfliktlinien durch demokratische Wahlen, in denen Wählerinnen und Wähler ihren - eher vermuteten - „Friedensinteressen“ hätten Ausdruck verleihen können, blieb aus (Manning 2004, S. 62-63, 83-84; vgl. auch Gromes 2003).

Auch mit Blick auf die 2010 abgeschlossenen Gacaca-Tribunale in Ruanda findet Susanne Buckley-Zistel (2005) kaum Hinweise auf eine Entfaltung der mit ihnen im Rahmen von Peacebuilding eigentlich beabsichtigten Wirkungen. Sie zeigt zudem auf, dass diese traditionelle Rechtsprechungsform, die eher auf einvernehmlichen Ausgleich denn auf Verurteilung und Bestrafung ausgerichtet ist, für die Aufarbeitung und Bewältigung des Genozids von vornherein nicht die Potenziale beinhaltet hat, um damit verbundene Wirkungserwartungen tatsächlich umsetzen zu können. Um nur einen von Buckley-Zistels Punkten herauszugreifen: Unter anderem sollten Gacaca-Tribunale, die jeweils auf der Ebene lokaler Gemeinden abgehalten wurden, zur Versöhnung (reconciliation) zwischen Tätern und Opfern des Genozids beitragen. Angeklagte Täter wurden dazu angehalten, ihre Beteiligung an Tötungen, Vergewaltigungen und/oder Plünderungen umfassend zu gestehen und um Vergebung zu bitten. Im Gegenzug sollte ihnen Strafminderung in Aussicht gestellt werden (Buckley-Zistel 2005, S. 5). Allerdings wurde bald deutlich, dass die meisten Täter dennoch keine umfassenden Geständnisse ablegten; zudem fanden sich unter Bedingungen anhaltender Unsicherheit nur wenige aussagewillige Zeugen. Darüber hinaus war das für die Aufarbeitung des Genozids veränderte Prozedere der Gacaca-Tribunale von vornherein so angelegt, dass es die Opfer erneut demütigen musste: Gewalt-Geständnisse in Anwesenheit der Opfer werden in Ruanda nicht als Grundlage für eine Entschuldigung, sondern - gerade auch von den Opfern selbst - vielmehr als öffentliche Bloßstellungen der Opfer aufgefasst (vgl. auch Brounéus 2010). Solche Gewalt-Geständnisse waren jedoch in der traditionellen Verhandlungspraxis von Gacaca-Tribunalen entsprechend gar nicht vorgesehen; in ihnen werden in Ruanda auf lokaler Ebene nicht Gewaltverbrechen, sondern vielmehr Familienstreitigkeiten oder Landkonflikte verhandelt (Buckley-Zistel 2005, S. 3-4).

Oliver Richmond und Jason Franks (2007) schließlich beschreiben, dass die mittlerweile jahrzehntelangen Peacebuilding-Bemühungen der „internationalen Gemeinschaft" in Kambodscha (seit 1991) - anders als über Peacebuilding beabsichtigt - mitnichten einen demokratischen Staat nach „westlichem “ Vorbild, sondern vielmehr einen „virtuellen Frieden“ hervorgebracht haben. Dieser zeichne sich dadurch aus, dass zwar Regierungsinstitutionen nach „westlichem“ Vorbild „aufgebaut“, die Praxis mehr oder weniger „freier“ Wahlen eingeführt und zudem mit internationaler Unterstützung „zivilgesellschaftliche“ Organisationen (lokale NGOs) hervorgebracht wurden. Das Problem: Sie basieren allesamt weniger auf liberal-demokratischen Prinzipien, sondern vielmehr auf Logiken politischer und ökonomischer Patronage. Diese Entwicklung sei, so argumentieren 
Richmond und Franks, zum Teil sicherlich den sozio-kulturellen Gegebenheiten in Kambodscha, vor allem aber auch einem unüberlegten und teilweise eben über diese sozio-kulturellen Gegebenheiten uninformierten Agieren kritikresistenter internationaler Akteure geschuldet (Richmond u. Franks 2007, S. 34-44). Um nur einen von zahlreichen Punkten aus der Untersuchung beispielhaft herauszugreifen: Die in Kambodscha nach „westlichem“ Vorbild organisierte lokale NGOSzene, deren Korruptheit und Einbindung in politische und ökonomische Patronage-Netzwerke heute von internationalen Akteuren beklagt wird, ist überhaupt erst im Zuge von Bemühungen entstanden, über finanzielle Unterstützung für NGO-förmige Organisationen eine Zivilgesellschaft nach „westlichem“ Vorbild zu schaffen. Richmond und Franks konstatieren: „The lessons from Cambodia seem to be that liberal peacebuilding either may take much longer to install than generally believed by international actors or, more negatively, is conceptually flawed or locally unsuited“ (Richmond u. Franks 2007, S. 45).

Der zentrale Beitrag, den diese und ähnliche Arbeiten leisten (vgl. etwa auch Chandler 2000; Shaw 2007), besteht daher vor allem darin, dass sie das Auseinanderklaffen zwischen Anspruch und Wirklichkeit (Norm und Praxis) im Peacebuilding aufarbeiten und so zudem überhaupt erst konkrete Bilder davon liefern, wie Peacebuilding-Ergebnisse empirisch tatsächlich „,aussehen“. Sie haben zudem gemeinsam, dass sie die Oberflächlichkeit von Peacebuilding-Ergebnissen in erster Linie auf die Fehler externer Peacebuilding-Akteure zurückführen. Lokale Akteure werden dabei tendenziell als missverstandene Empfänger thematisiert, für die zukünftig lediglich besseres Peacebuilding entwickelt werden müsse.

\subsection{Unter der Oberfläche: Peacebuilding als Interaktion}

Im Gegensatz zu den auf projekt- oder akteursspezifische Oberflächendefekte fokussierten Analysen vollziehen Michael Barnett und Michael Zürcher (2009) einen deutlichen Perspektivenwechsel: Sie formulieren ein akteursorientiertes Modell, in dem die Empfänger von Peacebuilding mit den internationalen Akteuren von Peacebuilding in strategische Interaktionen treten. Aus dieser Perspektive wird es überhaupt erst möglich, das Entstehen oberflächlicher Ergebnisse nicht als externes Versagen, sondern als Resultat eben solcher Interaktionsprozesse zwischen internationalen und lokalen Akteuren zu verstehen.

Modelltheoretisch gehen Barnett/Zürcher dabei von drei Typen relevanter Akteure aus, deren strategische Interaktionen die Ergebnisse von Peacebuilding prägen: erstens von internationalen „peacebuilders“, die stabile und demokratische staatliche Institutionen aufbauen wollen, ihren Fokus aber im Zweifelsfall eher auf Stabilität bzw. Sicherheit (Nicht-Krieg) legen; zweitens von (formal-)staatlichen Eliten im Nachkriegsland, die in erster Linie an der Macht bleiben und ihre Macht nach Möglichkeit im Zuge von Peacebuilding weiter festigen oder gar ausbauen wollen; und drittens von subnationalen Eliten im Nachkriegsland, die danach streben, möglichst unabhängig von staatlichen Eliten zu bleiben und ihre Machtbasen abseits der Hauptstadt zu erhalten bzw. sie im Zuge von Peacebuilding weiter zu festigen und auszubauen (Barnett u. Zürcher 2009, S. 31-32). Alle drei Typen werden dabei aus Gründen der Komplexitätsreduktion als „einheitli- 
che Akteure" konzipiert. Um sich für die Modellbildung auf die strategischen Interaktionen zwischen den Akteurs-Typen konzentrieren zu können, klammern Barnett und Zürcher somit Wert- und Interessen-Konflikte, Koordinationshindernisse oder auch potenzielle Informationsasymmetrien zwischen den verschiedenen Akteuren der „internationalen Gemeinschaft“ auf der einen Seite, zwischen rivalisierende Parteien, Gruppen und mächtigen Einzelpersonen innerhalb von staatlichen und subnationalen Eliten auf der anderen Seite, bewusst aus ihren Überlegungen aus (Barnett u. Zürcher 2009, S. 29).

Als Produkte der Interaktionen zwischen den drei Akteurs-Typen sind in dem Modell dann grundsätzlich vier verschiedene Peacebuilding-Ergebnisse denkbar: kooperatives, eingeschränktes ( „compromised“), konfrontatives und „captured“ Peacebuilding. Das aus Sicht internationaler „peacebuilders“ optimale, allerdings zugleich wenig wahrscheinliche Ergebnis wäre „kooperatives Peacebuilding“, in dem lokale Eliten die Programme internationaler „peacebuilders“ uneingeschränkt übernehmen würden (Barnett u. Zürcher 2009, S. 33). Das zweitbeste Ergebnis wäre „eingeschränktes Peacebuilding“, in dem internationale „peacebuilders" und lokale staatliche und subnationale Eliten für alle Seiten tragbare Kompromisse aushandeln würden: Barnett und Zürcher nehmen dabei an, dass „peacebuilders“ grundsätzlich bereit sind, Abstriche an demokratisierenden, strukturellen Reformen zu machen, wenn lokale Eliten ihnen im Austausch für ein weniger ambitioniertes Peacebuilding Stabilität und Legitimität und die Möglichkeit zusichern, einige ihrer Maßnahmen auf den Weg zu bringen und zudem Erfolge präsentieren zu können:

„They [„peacebuilders“, Anm. SC/AM] have developed a culture of principled pragmatism, ready to make compromises in the face of hard realities. They have an organizational interest in demonstrating success, especially once they have committee resources to the operation. Finally, they know the preference rankings of state elites and thus can anticipate that if they defect and attempt to revise the bargain then state elites are likely to resist“" (Barnett u. Zürcher 2009, S. 34).

Lokale Eliten (staatlich und subnational) wiederum würden ihren Machterhalt von „eingeschränktem Peacebuilding“ nicht weiter gefährdet sehen, weil darin schließlich gerade von jenen strukturellen Reformen Abstand genommen werden müsste, die ihren Machterhalt unter Umständen gefährden könnten. Da „eingeschränktes Peacebuilding “ insofern für alle beteiligten Akteure grundsätzlich zustimmungsfähig sein dürfte, kommen Barnett und Zürcher zu dem Schluss, dass es vermutlich das beste Ergebnis ist, das internationale „peacebuilders“ in den meisten Fällen erreichen können: „Compromised peacebuilding, therefore, becomes the equilibrium outcome because the parties have little incentive to defect" (Barnett u. Zürcher 2009, S. 34).

Auf dem dritten Rang in der Präferenzordnung internationaler "peacebuilders“ landet „konfrontatives Peacebuilding“. Dieses Ergebnis entstünde dann, wenn lokale Eliten externe Programme nicht unverändert übernehmen würden und es zudem nicht gelänge, einen tragfähigen Kompromiss auszuhandeln. Stattdessen würden lokale Eliten den „peacebuilders“ aktiv Widerstand entgegensetzen, so dass diese sich gezwungen sähen, ihre Peacebuilding-Bemühungen auszusetzen 
(Barnett u. Zürcher 2009, S. 33). Aus Sicht internationaler „peacebuilders“ das schlechteste und aber zugleich aus Sicht lokaler Eliten das beste Ergebnis bestünde dann in der vierten Variante des „,eroberten Peacebuilding“: Bei diesem Ergebnis würde es lokalen Eliten gelingen, die Aktivitäten der „peacebuilders“ de facto unter ihre Kontrolle zu bringen. Barnett und Zürcher argumentieren dabei, dass es vermutlich eher subnationalen als staatlichen Eliten gelingen kann, dieses Ergebnis zu erreichen. „Peacebuilders“ seien in Nachkriegsländern nun einmal gerade außerhalb der Hauptstädte auf lokale Unterstützung angewiesen. Subnationale Eliten, deren Machtbasen außerhalb der Hauptstädte in für externe „peacebuilders“ in jeder denkbaren Hinsicht „unwegsamem Gelände“ liegen, hätten insofern von vornherein eine bessere Verhandlungsposition:

\begin{abstract}
„[...] in comparison to state elites, subnational elites might have greater bargaining leverage. Peacebuilders are increasingly and notoriously out of their depths the further they get away from the capital city, tend to be more isolated and thus more dependent on subnational elites to provide security, and are more dependent on subnational elites to provide critical information and protection " (Barnett $\mathrm{u}$. Zürcher 2009, S. 36).
\end{abstract}

Barnett und Zürcher illustrieren dieses Argument unter anderem am Beispiel von Afghanistan, wo staatliche Eliten nach 2002 lediglich „eingeschränktes Peacebuilding " erreicht haben, während subnationale Eliten, die sich weitab von Kabul als unverzichtbare Helfer auf der Jagd nach Taliban und Al-Quaeda-Kämpfern präsentierten, teilweise „erobertes Peacebuilding “ erringen konnten (Barnett u. Zürcher 2009, S. 42-43). Allerdings: Genau genommen scheint diese Illustration schon über das von Barnett und Zürcher formulierte Modell hinauszugehen es sei denn, die Jagd auf Taliban und Al-Quaeda-Kämpfer würde als integraler Bestandteil von Peacebuilding verstanden, was bei Barnett und Zürcher jedoch offensichtlich nicht der Fall ist (Barnett u. Zürcher 2009, S. 47). Die Möglichkeit von politischen Agenden, die „peacebuilders“ von Peacebuilding ablenken könnten - eben beispielsweise der „Krieg gegen den Terror“ - ist in der für das Modell konstruierten Präferenzordnung der „peacebuilders“ ansonsten zumindest nicht explizit vorgesehen.

\title{
4. Lokale Ownership und Koordination und Integration
}

Angesichts der Oberflächlichkeit bisheriger Peacebuilding-Ergebnisse machen sich zumindest in Teilen des akademischen Peacebuilding-Mainstreams Ernüchterung und die Überzeugung breit, dass ein erster Schritt hin zu der Verringerung dieser Oberflächlichkeit im „Herunterschrauben“ der Erwartungen an Peacebuilding bestehen könnte (vgl. etwa Manning 2004, S. 83-84; Barnett u. Zürcher 2009, S. 48; Paris u. Sisk 2009b, S. 311). Dabei müsste das Ziel tiefgreifender Veränderungen, die dauerhaften und selbsttragenden Frieden ermöglichen sollen, nicht aufgegeben werden; aber es müssten auch Teilerfolge als Erfolge anerkannt werden können. In diesem Sinne unterscheiden etwa Call und Cousens (2008) zwischen maximalistischen, minimalistischen und moderaten Erfolgskriterien für Peacebuilding. Während die maximalistische Variante auf die Überwindung der 
ursprünglichen Kriegsursachen („root causes“) und die Herstellung demokratischer Staatlichkeit zielt, wäre das minimalistische Kriterium schon dann erreicht, wenn kein Rückfall in kriegerische Gewalt stattfände. Den Vorzug geben Call und Cousens allerdings einem moderaten Kriterium, demzufolge der Erfolg von Peacebuilding darin bestünde, dass ein Rückfall in kriegerische Gewalt vermieden und gleichzeitig eine „annehmbare Regierungsform“ („decent governance“) erreicht würde, die ein - nicht genauer definiertes - Mindestmaß an politischen Rechten und Partizipationsmöglichkeiten zulassen müsste: „This moderate standard is pragmatically and normatively appealing, though it is important to acknowledge that it, too, is imperfect, difficult to quantify, and leaves important issues about governance comparatively underexamined" (Call u. Cousens 2008, S. 8). Im Wesentlichen soll über ein solches moderates Kriterium anerkannt werden, dass selbst oberflächliche Ergebnisse wie etwa „eingeschränktes Peacebuilding“ (Barnett u. Zürcher 2009, S. 34) besser seien als vieles andere, was Bevölkerungen ohne Peacebuilding hätte passieren können. Im Rahmen auch solcher Ergebnisse würde von lokalen Eliten schließlich zumindest formal die Legitimität demokratischer und rechtstaatlicher Normen anerkannt, wodurch sich wiederum mittelund langfristig Wege für Wandel eröffnen könnten: „They [demokratische und rechtstaatliche Normen und Symbole, Anm. SC/AM] can become public commitments that even hypocritical reformers must take into account. They can be used by local and international reformers to continue to press for change " (Call u. Cousens 2008, S. 48; vgl. auch Barnett u. Zürcher 2009, S. 48). Ob und wann es dazu tatsächlich kommt, bleibt jedoch offen. Ebenso gut denkbar wäre, dass im Zuge oberflächlich implementierter Rechtstaatlichkeit und einer eben nur symbolischen Übernahme demokratischer Praktiken stabile und international anerkannte - wohlmöglich sogar als Partner geschätzte - autoritäre Regime entstehen.

Es gibt im akademischen Mainstream angesichts bisheriger Oberflächlichkeit jedoch auch die genau gegensätzliche Reaktion, dass die Messlatte für Peacebuilding-Erfolge erst recht hoch gehalten werden müsse. Dies ist beispielsweise in den Beiträgen des von Baranyi (2008a) herausgegebenen Sammelbands der Fall, deren gemeinsame Forderung im abschließenden Kapitel wie folgt zusammengefasst wird:

„What seems possible - namely, stabilization - is not sustainable and therefore, not desirable. What seems impossible - namely sustainable peace - may be possible with more imagination and innovation on the part of national actors as well as greater humility and long-term engagement on the part of international actors" (Baranyi u. Powell 2008, S. 314).

Ob die Messlatte nun niedriger gehängt oder hoch gehalten werden soll, Überlegungen im Hinblick auf die Möglichkeit einer Steigerung der „Tiefenwirksamkeit" von Peacebuilding kreisen vor allem um zwei (komplementäre) Umsetzungsprinzipien, die in der politischen Praxis längst offiziell anerkannt sind und Leitbilder für aktuelle Reformen darstellen. Da wäre zum einen das Prinzip einer konsequenten Herstellung lokaler Ownership für Peacebuilding-Maßnahmen, zum anderen das Leitbild „Koordination und Integration“ zwischen den an Peacebuilding-Maßnahmen beteiligten Akteuren. Beide Strategien werden zwar im 
akademischen Mainstream bereits in neue Empfehlungsformeln gegossen, aber auch hinsichtlich ihrer bisherigen praktischen Umsetzung mit erheblicher Skepsis betrachtet. Die wohl nicht unberechtigte Frage lautet, ob es sich bei lokaler Ownership und „Koordination und Integration“ nicht um bedeutungsarme Klischees handelt, die für sich genommen noch keinen besonderen Wert darstellen.

\subsection{Lokale Ownership und das Ringen um praktische Bedeutung}

Wenn über Peacebuilding diejenigen Machtkonstellationen, Herrschaftsverhältnisse und Lebensweisen verändert werden sollen, die - jeweils aus Sicht der externen Akteure - für die Dynamik gewaltsamer Konfliktspiralen in der Vergangenheit verantwortlich gemacht werden, dann würde es ohne Frage die „Tiefenwirksamkeit“ von Peacebuilding steigern, wenn die jeweils betroffenen Bevölkerungen sich die extern verschriebenen Maßnahmen zu eigen machen würden und diese Veränderungen selbst bewirken könnten. Paradoxerweise stellt sich dann allerdings die Frage: Wie ließe sich eigentlich sicherstellen, dass lokale Akteure sich Peacebuilding-Maßnahmen zu Eigen machen und so die erwünschte „Tiefenwirksamkeit“ von Peacebuilding hervorbringen? Und wie könnte lokale Ownership überhaupt möglich sein, wenn lokale Akteure die Ziele von Peacebuilding schlicht nicht teilen?

Auf der Suche nach der praktischen Bedeutung von lokaler Ownership zeichnet Simon Chesterman (2007) nach, dass das Konzept Mitte der 1990er-Jahre zunächst vor allem im Kontext von „Entwicklungszusammenarbeit“ an Prominenz gewann (vgl. auch Reich 2006, S. 5). Ausgehend von einem Strategie-Dokument des Development Assistance Committee (DAC) der OECD aus dem Jahr 1995 wurde fortan von immer mehr Akteuren immer häufiger postuliert, dass die Bevölkerungen in Entwicklungsländern die „owners“ von Entwicklungspolitiken werden müssten, wenn diese Politiken zukünftig mehr Aussicht auf Erfolg haben sollten. Dabei wurde allerdings stets offen gelassen, wie genau ihre „Eigentümerschaft" zu operationalisieren wäre (vgl. Chesterman 2007, S. 7). Daran änderte sich auch nichts, nachdem lokale Ownership als Umsetzungsprinzip für Peacebuilding entdeckt worden war. Chesterman illustriert dies am Beispiel eines Berichts des ehemaligen UN-Generalsekretärs Kofi Annan aus dem Jahr 2004. Darin heißt es: "[...] we must learn better how to respect and support local ownership, local leadership and a local constituency for reform, while at the same time remaining faithful to United Nations norms and standards." (Annan 2004, zitiert in Chesterman 2007, S. 10). Die Frage, wie genau dies in Nachkriegsländern möglich sein soll, in denen UN-Normen aber nun einmal verletzt und UN-Standards nicht erfüllt werden, bleibt bislang unbeantwortet (Chesterman 2007, S. 11). Dennoch wurde lokale Ownership von UN, OECD, EU und auch von der Bundesregierung zum grundlegenden Umsetzungsprinzip für Entwicklungspolitiken und Peacebuilding erklärt (vgl. Major et al. 2011, S. 11).

Die Kritik aus dem akademischen Mainstream fällt zumindest stellenweise entsprechend harsch aus: Lokale Ownership sei ein letzten Endes nicht nur bedeutungsleeres, sondern sogar irreführendes Klischee, welches genutzt werde, um ein nicht näher definiertes Ausmaß an lokaler Kontrolle über Peacebuilding-Maßnah- 
men zu implizieren, das bei der Umsetzung von Peacebuilding jedoch nie auch nur ansatzweise realisiert werde (Chesterman 2007, S. 20; vgl. ähnlich Reich 2006, S. 14). Und in der Tat bedeutet die Herstellung lokaler Ownership praktisch allenfalls ein Hinzuziehen lokaler Akteure im Sinne von mehr oder weniger institutionalisierten und meist nicht bindend entscheidungsrelevanten Beratungen ${ }^{12}$ - aber gerade eben nicht, dass Peacebuilding tatsächlich in dem Sinne an lokale Akteure übergeben wird, dass sie selbst die Inhalte und die Umsetzung von PeacebuildingMaßnahmen bestimmen. Eine aus Sicht internationaler Akteure unfreiwillige Ausnahme mag das weiter oben nach Barnett und Zürcher (2009) beschriebene „eroberte Peacebuilding “ darstellen, in dem insofern Ownership entsteht, als lokale Akteure, die zudem die Ziele von Peacebuilding nicht teilen, ein erhebliches Maß an Kontrolle über Peacebuilding-Aktivitäten erringen - mit entsprechend deutlich negativen Konsequenzen für die „Tiefenwirksamkeit“ von Peacebuilding.

Dennoch wird das Konzept lokaler Ownership auch im akademischen Peacebuilding-Mainstream nicht verworfen. Vielmehr wird die praktische Herausforderung darin gesehen, für unterschiedliche Phasen einer Intervention und für unterschiedliche Peacebuilding-Maßnahmen jeweils das „richtige Maß“ an lokaler Ownership herzustellen. Dieses „richtige Maß“ würde sich ohnehin keinesfalls durch eine vollständige Übergabe von Peacebuilding-Maßnahmen an lokale Akteure auszeichnen, sondern dadurch, dass die angestrebten Peacebuilding-Ergebnisse unter „Beteiligung“ lokaler Akteure, welche die grundsätzlichen Ziele von Peacebuilding teilen, auf Umsetzungsarten und in Ergebnisformen erreicht werden, die lokalen Bedürfnissen entsprechen, als legitim akzeptiert werden und entsprechend „tiefenwirksam“ bzw. von Dauer sein könnten (vgl. etwa Chesterman 2007, S. 20-21; Campbell 2008, S. 564; Narten 2009, S. 252; Paris u. Sisk 2009b, S. 307-308). Ein ungefähres Konzept dazu, wie eine solche „Beteiligung“ in der Praxis auszusehen hätte, ist bei Jens Narten (2009) zu finden. Er argumentiert, es sei notwendig, lokale Akteure nach und nach umfassend in alle Phasen und Aspekte der Intervention einzubeziehen; in die Planung, Umsetzung und Evaluation von Peacebuilding-Maßnahmen:

„Within this framework, a 'high level' of local ownership is the point at which external statebuilders have effectively included local stakeholders at all levels [...] meaning that local actors have been consulted and included in the problem and needs assessment, project design, and implementation as well as the evaluation and control of the overall process and its outcome" (Narten 2009, S. 254; Hervorhebung SC/AM).

12 Bei den so hinzugezogenen lokalen Akteuren handelt es sich neben hochrangigen Politikern und Beamten häufig um Angehörige „zivilgesellschaftlicher“ Organisationen, insbesondere lokaler NGOs, von denen angenommen wird, dass gerade sie die Ziele von Peacebuilding teilen - bzw. die sich häufig erst mit finanzieller Unterstützung durch externe Akteure als ,zivilgesellschaftliche“ Interessenvertreter für Peacebuilding-Anliegen konstituieren. Während diese Möglichkeit finanzieller Unterstützung qua Peacebuilding-befürwortender NGO-Tätigkeit in Nachkriegsländern, in denen lukrative und statuserhöhende ökonomische Gelegenheiten meist für den Großteil der Bevölkerung rar sind, häufig geradezu einen NGO-Boom auslöst, werden nicht-NGO-förmige lokale Organisationen von externen Akteuren häufig gar nicht erst als förderungswürdig bzw. überhaupt nicht (an-)erkannt (vgl. Reich 2006, S. 13-16; Jarstad 2008a, S. 127; Richmond u. Franks 2007, S. 39; Stromseth et al. 2006, S. 342). 
In eine ähnliche Richtung gehen auch die Überlegungen von Hannah Reich (2006). Sie plädiert zudem dafür, den Begriff lokaler Ownership ganz aufzugeben und stattdessen in den Prozessen von Planung und Umsetzung gemeinsame „Lernfelder" zu ermöglichen. In diesen, sollten externe und lokale Akteure sich nicht nur über die Inhalte und Umsetzungsmodalitäten von Peacebuilding austauschen, sondern auch die asymmetrischen Beziehungen zwischen ihnen, in denen lokalen Akteuren „Beteiligung“ stets nur in einem begrenzten Maß gewährt wird, offen thematisieren und problematisieren (Reich 2006, S. 23-24).

\section{2 „Koordination und Integration“: Klischee oder Irrweg?}

Ebenso wie lokaler Ownership haftet „Koordination und Integration“ der Status eines Klischees (Jennings u. Kaspersen 2008, S. 443) oder Mantras (Paris 2009, S. 52) an; und das, obwohl „Koordination und Integration“ seit Ende der 1990erJahre das Leitbild für Reformen darstellen, die sowohl innerhalb des UN-Systems (vgl. Campbell u. Kaspersen 2008, S. 471-475) ${ }^{13}$ als auch auf nationalstaatlicher Ebene unternommen worden sind, um die Effizienz und Effektivität von Peacebuilding-Bemühungen zu steigern (vgl. Bendix u. Stanley 2008, S. 14-16). ${ }^{14}$

$\mathrm{Zu}$ analytischen $\mathrm{Zwecken}$ lassen sich die Funktionen von „Koordination“ einerseits und „Integration“ andererseits, durchaus unterscheiden: Bei Koordination geht es primär darum, Strukturen und Abläufe zu schaffen, die es Peacebuilding-Akteuren ermöglichen, sich je fallbezogen untereinander auf operative Ziele und Strategien für Peacebuilding zu verständigen, aber auch innerhalb relevanter Ministerien, Agenturen, Unterorganisationen gemeinsame Ziele und Strategien zu formulieren. Dahinter steht in der Regel das Ziel, Doppelungen und Widersprüche zu vermeiden, also die Effizienz von Peacebuilding-Bemühungen zu steigern. Integration hingegen wäre vor allem darauf ausgerichtet, Strukturen und Abläufe schaffen, in denen Peacebuilding-Maßnahmen wirkungsoptimierend aufeinander abgestimmt werden könnten, um die Effektivität von Peacebuilding zu steigern. Dabei müssten dann zudem zukünftige Probleme hinsichtlich der Wirkung von Peacebuilding antizipiert und bewältigt werden. Sowohl in den politischen Reformbeschlüssen als auch in der Diskussion von „Koordination und Integration“ im akademischen Mainstream werden beide Begriffe jedoch meist synonym verwendet bzw. in manchen Arbeiten wird vor allem der Begriff Koordination verwendet (Paris 2009), während in anderen überwiegend von Integration gesprochen wird (Campbell 2008). Mit Blick auf die Reformbemühungen der UN, auf die sich die im Folgenden diskutierten Arbeiten beziehen, weist Susanna Campbell

13 Die hier im Weiteren zitierten Beiträge von Jennings und Kaspersen, Campbell und Kaspersen und Campbell (alle 2008) stammen aus einer Ausgabe von International Peacekeeping, die insgesamt den Reformen im UN-System gewidmet ist.

14 In Deutschland laufen diese Reformbemühungen seit 2004 unter dem Titel „Aktionsplan ,Zivile Krisenprävention, Konfliktlösung und Friedenskonsolidierung““ (vgl. Stengel u. Weller 2010, S. 96-97). 2010 hat die Bundesregierung den mittlerweile dritten Bericht zum Stand der Umsetzung ihrer Integrations- und Koordinationsprojekte vorgelegt. Ein durchweg kritischer Kommentar zu diesem Bericht ist auf der Internetseite der „Plattform Zivile Konfliktbearbeitung“ zu finden (http://www.konfliktbearbeitung.net/node/3765; zugegriffen 18.05.2011). 
zudem darauf hin, dass ohnehin auch die zugrundeliegenden Reformbeschlüsse keine genauen Definitionen dieser Begriffe enthalten (Campbell 2008, S. 557). Worum es geht, lässt sich dennoch einigermaßen klar ableiten:

„The goals $[. .$.$] are to increase the efficiency and effectiveness of the UN system ope-$ rating alongside or as part of multidimensional peace operations. The objectives - to consolidate country-level leadership, centralize support from headquarters, systematize joint planning, institute interagency programming, and develop compacts between the UN system and the national government [des jeweiligen PeacebuildingEmpfängerlands, Anm. SC/AM] - are assumed to be means to these ends" (Campbell u. Kaspersen 2008, S. 475).

Die Skepsis, die Integrations- und Koordinations-Reformen entgegengebracht wird, bezieht sich nicht nur auf ihre als schleppend und hindernisbehaftet diagnostizierte Umsetzung (vgl. Jennings u. Kaspersen 2008, S. 444; Campbell 2008, S. 560). Darüber hinaus werden grundsätzliche Zweifel daran formuliert, inwieweit solche Reformen tatsächlich zur Steigerung der Effizienz und Effektivität („Tiefenwirksamkeit“) von Peacebuilding beitragen könnten. Aus einer solchen, grundsätzlich skeptischen Perspektive argumentiert Roland Paris (2009), dass mangelnde Koordination bei der Umsetzung von Peacebuilding schlichtweg nicht das einzige zentrale Problem sei: Zwar gäbe es in der Tat Doppelungen, Widersprüche und Unklarheiten in Peacebuilding-Bemühungen; aber Doppelungen, Widersprüche und Unklarheiten seien mitnichten ausschließlich auf Koordinationsprobleme zurückzuführen (Paris 2009, S. 55). Stattdessen würden sie vor allem auch daraus resultieren, dass verschiedene internationale Organisationen, UNUnterorganisationen, staatliche Akteure etc. ihre Peacebuilding-Bemühungen jeweils mit unterschiedlichen und zum Teil widersprüchlichen Philosophien und operativen Zielen umsetzen - woran auch Koordination aller Voraussicht nach nichts ändern könnte: „While most international actors subscribe to the broad goals of transforming war-torn states into liberal market democracies, there is no universal agreement on what is required to achieve this goal, or how to achieve it under different circumstances" (Paris 2009, S. 59). In den bisherigen Reformbemühungen würde dieses grundsätzliche Problem nicht ausreichend reflektiert, weshalb letztlich unrealistische Erwartungen an ihre Problemlösungspotenziale gestellt würden (Paris 2009, S. 59-60; vgl. ebenso Campbell 2008, S. 561). Des Weiteren müsse in den dennoch dringend notwendigen Koordinationsreformen darauf geachtet werden, keine „falsche Art“ von Koordination zu installieren, nämlich zentralisierte und hierarchisierte Koordination, welche die Flexibilität von Peacebuilding-Akteuren einschränken und es ihnen letztlich erschweren würde, auf veränderte Bedingungen und Anforderungen in kaum berechenbaren Nachkriegssituationen zu reagieren. Eine solche Reaktionsfähigkeit sei auch deshalb von zentraler Bedeutung, ,[...] because international peacebuilding agencies 
have only limited knowledge of what is required to succeed in the ambitious task of stabilizing a fragile country after war" (Paris 2009, S. 63). ${ }^{15}$

Insbesondere die Notwendigkeit, solches „Nichtwissen“ in der Planung von Koordinations- und Integrationsreformen zu berücksichtigen, wird auch von Campbell hervorgehoben. Sie wendet ein, dass unter Bedingungen von „Nichtwissen" (wobei sie diesen Begriff selbst nicht nutzt) beispielsweise gerade die Doppelung von Peacebuilding-Maßnahmen durchaus wünschenswert sei, da im Vorhinein kaum antizipiert werden könne, welche Maßnahme letzten Endes tatsächlich Wirkung zeigen werde:

„,[...] the assumption that the duplication of activities is ineffective may not be wholly accurate. Because the trajectory of war-to-peace transitions is unpredictable, and it is difficult to know which activities will lead to the desired impact, some degree of duplication may be necessary. If the same problem is approached from different directions, there is a greater probability that it will be solved" (Campbell 2008, S. 563).

Um die Effektivität von Peacebuilding zu erhöhen, sei es entsprechend notwendig, zum einen Wissen über die Wirkungen von Peacebuilding zu erarbeiten und zum anderen anzuerkennen, dass Peacebuilding-Akteure, um tatsächlich „Frieden " fördern zu können, eigentlich darauf vorbereitet und organisationell darauf ausgerichtet sein müssten, in veränderten Kontexten je aufs Neue zu entdecken, ob ihre Maßnahmen tatsächlich Frieden fördern - und wie reagiert werden kann, falls dies nicht der Fall sein sollte. Campbell räumt zudem ein, dass gerade eine solche Anerkennung der Komplexität und Unberechenbarkeit von Friedensprozessen innerhalb der UN nicht weit verbreitet sei (Campbell 2008, S. 563-564).

\section{5. „Nichtwissen“ und der Weg in die Sackgasse}

"How do we know that any self-described peacebuilding instrument/initiative even works, aside from listening to anecdotal stories shared over warm beer in generic bars in war-prone regions around the world?" (Bush 2003, S. 46). Diese bereits vor einigen Jahren von dem Friedens- und Konfliktforscher Kenneth Bush zugespitzt formulierte Frage hat bis heute nichts von ihrer Aktualität verloren. Zwar deuten die quantitativ-vergleichend erarbeiteten Korrelationen zwischen Peacebuilding und Nicht-Krieg (siehe oben) durchaus an, dass es Wirkungen gibt und dass sie zumindest grob in die angepeilte Richtung verlaufen. Ob dies aber tatsächlich der Fall ist und - wenn ja - wie genau und womöglich um den Preis welcher nicht-intendierten Wirkungen, bleibt größtenteils im Dunkeln. In den Arbeiten des akademischen Mainstreams wird entsprechend darauf hingewiesen, dass es notwendig sei, genauere Einsichten in die Wirkungen von Peacebuilding zu erlangen: „[...] the evidence remains largely correlative rather than causal and therefore wanting for finer-grained analysis of causality and impact." (Call u.

15 In welchem Ausmaß solche Flexibilität und Reaktionsfähigkeit aktuell überhaupt gegeben ist und insofern durch Reformbemühen gefährdet sein könnte, ist allerdings durchaus fraglich (vgl. Campbell 2008, S. 561). 
Cousens 2008, S. 5; vgl. auch Campbell 2008, S. 363; Paris u. Sisk 2009, S. 309). Ein eben solches kausales Wissen über spezifische Wirkungszusammenhänge bräuchte es in der Tat auch, um über Strategien wie lokale Ownership oder „Koordination und Integration “ die Wirksamkeit von Peacebuilding zu erhöhen, bzw. um mit den intendierten wie nichtintendierten (Aus-)Wirkungen von Peacebuilding-Maßnahmen umgehen zu können.

Allerdings ist jede Wirkungsanalyse, die sich komplexer und dynamischer sozialer Realität stellt, die nicht stabil gehalten werden kann, zwangsläufig mit folgenschweren methodologischen Problemen konfrontiert; dies gilt selbstverständlich auch für Analysen der Wirkungen von Peacebuilding. So lassen sich Aspekte sozialer Realität bzw. Formen von sozialem Wandel (etwa eine „Tiefenwirkung“ von Peacebuilding), die anhand zuvor festgelegter, analytischer Kriterien als intendierte Wirkungen ausgemacht werden, kaum einzelnen Faktoren - hier Peacebuilding-Maßnahmen - kausal zuordnen. Für das, was als intendierte Wirkung von Peacebuilding erscheint, lässt sich entsprechend nie ausschließen, dass es zumindest teilweise oder auch gänzlich durch Ereignisse und/oder Prozesse ausgelöst bzw. hervorgebracht wurde, die nur indirekt oder auch gar nicht mit der Umsetzung einer jeweiligen Peacebuilding-Maßnahme zu tun haben (vgl. Smith 2004, S. 59-60; Körppen 2007, S. 30-31; Quack 2007, S. 36-37).

Humphreys und Weinstein (2007) haben diesen Umstand in einer vergleichsweise aufwendigen Studie über das gemeinhin als erfolgreich (im Sinne von intendiert wirksam) geltende DDR-Programm in Sierra Leone eindrucksvoll demonstriert: In ihrer Studie untersuchen sie auf der Basis von Umfragedaten, die unter insgesamt 1043 Ex-Kombattanten erhoben wurden, inwieweit diejenigen, die am DDR-Programm teilgenommen haben, als „besser reintegriert“ gelten können als diejenigen, die nicht am DDR-Programm teilgenommen haben bzw. die gar keinen Zugang zu dem Programm hatten. Im Ergebnis stellen Humphreys und Weinstein fest, dass sich entlang der von ihnen festgelegten Kriterien für Reintegrations-Erfolg kaum Unterschiede zwischen beiden Vergleichsgruppen feststellen lassen; beide erscheinen vielmehr in etwa gleich erfolgreich. Entsprechend bestehe eigentlich kein Grund zu der Annahme, dass gerade die (als solche definierten) Reintegrations-Erfolge derjenigen, die am DDR-Programm teilgenommen haben, auf Wirkungen des DDR-Programms zurückzuführen seien (Humphreys u. Weinstein 2007, S. 549-554). Ganz ausschließen lasse sich dies wiederum jedoch auch nicht - ebenso wenig wie deutliche Unterschiede im „Reintegrations-Erfolg“ der Vergleichsgruppen mit absoluter Sicherheit eine intendierte Wirksamkeit des DDR-Programms unter Beweis gestellt hätten (vgl. Humphreys u. Weinstein 2007, S. 560-561).

Ein weiteres methodologisches Problem besteht hinsichtlich der Erfassung nicht-intendierter Wirkungen interdependenter Entscheidungen: „Eine Intervention hat potentiell unbegrenzt viele und unbegrenzt komplexe Wirkungen, nämlich alle durch eine Intervention verursachten konfliktrelevanten Unterschiede zur (kontrafaktischen) Entwicklung ohne die Intervention“ (Quack 2007, S. 36-37). Aus dieser Masse lassen sich analytisch jedoch stets nur diejenigen Entwicklungen als mögliche Wirkungen herausfiltern, nach denen - anhand bestimmter, zuvor festgelegter Kriterien - tatsächlich auch gesucht wird. Insofern ist stets davon aus- 
zugehen, dass sich ein erheblicher Anteil der Wirkungen von Peacebuilding den jeweils angelegten Untersuchungskriterien entzieht. Dies betrifft insbesondere solche Wirkungen, die nicht beabsichtigt sind, von denen Forscherinnen und Forscher sich womöglich noch gar keine Vorstellungen machen können und nach denen entsprechend auch gar nicht erst gefragt wird. Darüber hinaus gilt selbstverständlich auch für jene Aspekte sozialer Realität bzw. für jene Formen von sozialem Wandel, die als nicht-intendierte Wirkungen von Peacebuilding definiert werden, das oben beschriebene Zuordnungsproblem.

Nicht zuletzt müsste Steuerungswissen über die Wirkungen von Peacebuilding zudem verlässliche Regeln dazu enthalten, unter welchen Bedingungen kausales Wirkungswissen zu bestimmten Peacebuilding-Maßnahmen (welches sich ohnehin nicht erarbeiten lässt), von seinen Erarbeitungskontexten auf bestimmte andere Kontexte übertragen werden kann; ob also beispielsweise davon ausgegangen werden könnte, dass eine nach Art des DDR-Programms in Sierra Leone geplante Maßnahme auch im Sudan erfolgreich sein würde. Da sich in komplexer sozialer Realität jedoch nie alle tatsächlich relevanten Kontextbedingungen ermitteln lassen, sondern immer nur diejenigen, die anhand bestimmter (im besten Fall theoretisch abgeleiteter) Kriterien als relevant ausgemacht werden, ist auch die Erarbeitung verlässlicher Übertragungsregeln mehr als fraglich. Erschwerend kommt hinzu, dass auch Peacebuilding-Maßnahmen in verschiedenen Kontexten nie ganz dieselben sein können, selbst wenn sie auf absolut identischen Planungsvorlagen und Ressourcenausstattungen beruhen sollten (was selten bis nie der Fall sein dürfte). Zudem werden Peacebuilding-Maßnahmen auch aufseiten der internationalen Akteure von Menschen umgesetzt, die sich hinsichtlich Ausbildung, kulturellem Hintergrund, Arbeitsauffassung, Begeisterungs- und Empathiefähigkeit, Integrität und (kultureller) Kompetenz voneinander unterscheiden. Es ist davon auszugehen, dass auch solche Unterschiede bzw. die Art und Weise, wie sie zum einen untereinander und zum anderen in Relation zur lokalen Bevölkerung und/oder zu den jeweiligen Verhandlungseliten „funktionieren“, erheblichen Einfluss auf die Wirkungen von Peacebuilding-Maßnahmen nehmen (vgl. Rubinstein et al. 2008).

Die Einsicht, dass die grundlegenden methodologischen Probleme von Wirkungsanalysen auch für die Untersuchung der Wirkungen von Peacebuilding gelten, ist dabei keinesfalls neu und auch bereits in aller Deutlichkeit formuliert worden. So kommt etwa die 2004 veröffentlichte, so genannte Utstein-Studie, welche die Umsetzungs- und anschließende Evaluierungspraxis in insgesamt 336 norwegischen, deutschen, niederländischen und britischen Peacebuilding-Maßnahmen untersucht hat, unmissverständlich zu dem Schluss: „,...] in the current state of knowledge, assessing the peacebuilding impact of an individual project is impossible" (Smith 2004, S. 59).

Zusammenfassend lässt sich daher festhalten, dass weder Steuerungswissen für Peacebuilding-Maßnahmen zur Verfügung steht noch eine Aussicht darauf besteht, dass solches Steuerungswissen überhaupt erarbeitet werden kann. Die eigentlich naheliegende Konsequenz, der Idee einer Steuerbarkeit der Wirkungen von Peacebuilding in aller Deutlichkeit eine Absage zu erteilen, wird im akademischen Mainstream jedoch nicht explizit gezogen; auch wenn sie in den weiter 
oben diskutierten Problemen mit „Koordination und Integration“ (siehe 4.3) eigentlich bereits enthalten ist (vor allem bei Campbell 2008 und Paris 2009).

Dass es jedoch notwendig ist, die Idee einer Steuerbarkeit von PeacebuildingWirkungen explizit und in aller Deutlichkeit aufzugeben, um aus der gedanklichen Steuerungs-Sackgasse herauszufinden, lässt sich an den Empfehlungen verdeutlichen, die Paris und Sisk zur Effektivitätssteigerung von StatebuildingMaßnahmen formulieren (2009b, S. 306-311). In dem abschließenden Kapitel des von ihnen herausgegebenen Sammelbands zu Statebuilding sind sie darum bemüht, internationale Akteure dafür zu sensibilisieren, dass ihre Maßnahmen vermutlich nicht wie beabsichtig wirken werden. Diese Steuerungsprobleme sind ihrer Diagnose nach allerdings nicht darauf zurückzuführen, dass Steuerung per se nicht möglich ist, sondern vielmehr darauf, dass Statebuilding eine in sich widersprüchliche Unternehmung sei, die entsprechend widersprüchliche Ergebnisse hervorbringe, die sich allerdings „managen“ lassen würden - selbst wenn noch nicht ausreichend viel über sie gewusst werde (Paris u. Sisk 2009b, S. 309). Zum Zweck eines solchen Managements empfehlen Paris und Sisk, bei der Umsetzung von Statebuilding-Maßnahmen von vornherein auf „Dilemmata“ vorbereitet zu sein. Sie identifizieren dann insgesamt fünf sehr allgemein und auf hohem Abstraktionsniveau gehaltene „Dilemmata“, die sie in der bisherigen Forschung zur Umsetzung von Statebuilding-Maßnahmen ausmachen und die internationalen Akteuren als Orientierungspunkte für das Antizipieren von Problemen dienen sollen. Die ersten vier dieser Dilemmata („Footprint dilemmas“, „Duration dilemmas“, „Participation dilemmas“, „Dependency dilemmas“) lassen sich grob zu der Warnung zusammenfassen, dass tiefe und andauernde Eingriffe in Nachkriegsländer zwar nötig erscheinen mögen, um die Sicherheitslage zu verbessern, die Umsetzung von Friedensabkommen durchzusetzen und zentrale Maßnahmen auf den Weg zu bringen; zugleich würden sie jedoch als Risiken in sich bergen, dass staatliche Akteure schwach gehalten werden bzw. sich ihrer Verantwortung entziehen (können), dass dauerhafte Abhängigkeiten von internationaler Unterstützung entstehen und/oder dass die externen Eingriffe in der lokalen Bevölkerung zunehmend als illegitim wahrgenommen werden. Umgekehrt würden weniger tiefe und andauernde Eingriffe womöglich dazu führen, dass Sicherheitslagen nicht verbessert, die Umsetzung von Friedensabkommen nicht durchgesetzt und zentrale Maßnahmen nicht auf den Weg gebracht werden. Die fünfte Art von „Dilemma“, auf die Paris und Sisk aufmerksam machen, betrifft sowohl die organisationelle als auch normative Kohärenz von Statebuilding-Maßnahmen („Coherence dilemmas"): Koordination, verstanden als die Herstellung von kohärenten Maßnahmen zwischen und innerhalb der Akteure der „internationalen Gemeinschaft“ und unter Einbeziehung lokaler Akteure, müsse als schwierig und nicht immer zielführend antizipiert werden (vgl. Paris 2009; siehe auch oben 4.2). Auch sei normative Kohärenz kaum erreichbar: „Principles such as democratic accountability, national self-determination, the rule of law and good governance all tend to be compromised - to varying degrees - by the very fact of international intervention [...]“ (Paris u. Sisk 2009b, S. 309). Für das „Management“ dieser „Dilemmata" und somit zugleich zur Effektivitätssteigerung von Statebuilding empfehlen Paris und Sisk dann, sowohl die intendierten als auch die nicht-intendierten Wir- 
kungen der eingesetzten Maßnahmen unter Hinzuziehen von „deep local knowledge " (Paris u. Sisk 2009b, S. 311) sowohl vor als auch während ihrer Umsetzung genau zu prüfen. Angesichts von „Nichtwissen“ ist die entscheidende Frage jedoch, wie dies überhaupt praktisch möglich sein könnte.

\section{Schluss: Raus aus der Sackgasse}

Einleitend hat dieser Beitrag mit der von Paris (2010) angebotenen Situationsdefinition aufgemacht, der zufolge Peacebuilding heute an einem Scheideweg steht, an dem eine Entscheidung darüber zu treffen sei, ob Peacebuilding verworfen oder verbessert werden sollte. Wer Position zu dieser von Paris eingeforderten Entscheidung beziehen will, muss jedoch sehr viel grundsätzlicher der Frage nachgehen, ob die Forschung zu Peacebuilding überhaupt Ergebnisse liefert, auf deren Grundlage Empfehlungen für praktische Verbesserungen formuliert werden können. Die Antwort muss eher pessimistisch ausfallen. Es wird sich im akademischen Peacebuilding-Mainstream, dessen Forschungsagenda ganz auf Bemühungen um die Verbesserung von Peacebuilding ausgerichtet ist, dagegen gesträubt, die Konsequenzen aus eigentlich schon längst zur Verfügung stehenden Einsichten zu ziehen: dass eine Steuerung der Wirkungen von Peacebuilding unter Bedingungen von „Nichtwissen“ über diese Wirkungen nicht möglich ist und dass „Nichtwissen " zudem angesichts der methodologischen Probleme, vor denen jede empirische Wirkungsanalyse steht, kaum zu überwinden sein wird. In dem Bemühen, zu einer Verbesserung von Peacebuilding beizutragen, verharrt der akademische Peacebuilding-Mainstream so in einer Sackgasse - ist aber zugleich Profiteur dieser Situation, da ihm weder innerhalb noch außerhalb der Wissenschaft die Existenzberechtigung abgesprochen wird. Ein Weg, der zumindest ein gutes Stück weit aus dieser Sackgasse hinaus führen könnte, bestünde darin, „Nichtwissen“ in aller Deutlichkeit zum Ausgangspunkt der Entwicklung von Verbesserungsvorschlägen zu machen. Dies böte zudem die Chance aus einer weitgehend westlich dominierten, selbstreferenziellen Forschungs- und Legitimierungspraxis auszubrechen, die Perspektiven eher verengt (u. a. Festhalten am methodologischen Nationalismus), als dass sie ihre Konzepte „brücken- oder reisefähig“ macht.

„Nichtwissen“ stellt die Peacebuilding-Praxis in letzter Konsequenz vor die enorme Herausforderung, Arbeitsprozesse bzw. „Peacebuilding-Routinen“ einzuführen, obwohl die Wirkungen der zu diesem Zweck eingesetzten Maßnahmen eben weder vorhergesagt noch gesteuert werden können (vgl. auch Campbell 2008, S. 563). In einer Ausgabe der Berghof Handbook Dialogue Series, die passenderweise ebenfalls den Titel „Peacebuilding at the Crossroads“ trägt (Schmelzle u. Fischer 2009), umreißen die Praktikerinnen Ulrike Hopp und Barbara Unger die analytischen und organisationellen Dimensionen dieser Herausforderung anhand von zwei Fragen: „[...] we believe that our field should look closer at two questions: where does change need to happen, and how well are we - and our organisations - equipped to inspire that change?" (vgl. Hopp u. Unger 2009, S. 79). Explizit vor dem Hintergrund von „Nichtwissen“ interpretiert, ergeben sich allein aus diesen Fragen Aufgaben- und Reflexionsfelder für praxisbezogene Forschung und wissenschaftliche Politikberatung. 
Dabei geht es, erstens, um die Entwicklung von analytischen Strategien zur Definition von Fokuspunkten, die es Praktikerinnen und Praktikern ermöglichen würden, in sozialer Realität zu erkennen, ob unter Bedingungen von Peacebuilding in Nachkriegsländern tatsächlich ein „Mehr an Frieden“ entsteht. Nur wenn dies der Fall sein sollte, könnte davon ausgegangen werden, dass PeacebuildingMaßnahmen zumindest keinen Schaden anrichten und möglicherweise sogar in intendierter Weise wirken; sollte unter Bedingungen von Peacebuilding hingegen kein „Mehr an Frieden“ oder sogar ein „Weniger an Frieden“ entstehen, müsste in Erwägung gezogen werden, dass Peacebuilding-Maßnahmen nicht in intendierter Weise wirken. Als Basis für die Definition solcher Fokuspunkte bedürfte es klar definierter Friedensverständnisse, in denen Frieden nicht an der Herstellung vermuteter Bedingungen für Frieden (etwa demokratischer Staatlichkeit) festgemacht, sondern im alltäglichen Leben der jeweils unmittelbar betroffenen Bevölkerungen verankert würde (vgl. ähnlich Mitchell 2005, S. 4; Free 2010, S. 55). An Fokuspunkten, die auf solchen Friedensverständnissen basieren müssten, könnten dann zwar nicht die Wirkungen einzelner Peacebuilding-Maßnahmen, aber „the prospects of peace itself" (Smith 2004, S. 60) in den Blick genommen werden. Dazu nur ein Beispiel: Wenn unter einem „Mehr an Frieden“ etwa ein Abnehmen von alltäglichen Gewalterwartungen verstanden würde, müsste für die Definition von Fokuspunkten zunächst ausgemacht werden, in welchen situativen Kontexten und sozialen Beziehungen in einem jeweiligen Nachkriegsland Gewalterwartungen bestehen. An diesen Fokuspunkten würde sich dann über Zeit beobachten lassen (und auch hierzu könnten verschiedene, etwa an unterschiedliche Sicherheitslagen angepasste analytische Strategien formuliert werden), ob Gewalterwartungen unter Bedingungen von Peacebuilding tatsächlich abnehmen, gleich bleiben oder womöglich sogar zunehmen.

Die Durchführung solcher extrem komplexen und arbeitsintensiven analytischen Tätigkeiten im Zuge der Umsetzung von Peacebuilding ist dabei überhaupt nur unter den Bedingungen vorstellbar, dass Praktikerinnen und Praktiker gezielt für sie ausgebildet würden und dass diese analytischen Tätigkeiten zu festen Bestandteilen von Peacebuilding-Routinen gemacht würden. Letzteres müsste dann sinnvollerweise auch beinhalten, dass organisationelle Strukturen und Prozesse so gestaltet würden, dass in ihnen tatsächlich flexibel auf die Ergebnisse der analytischen Tätigkeiten reagiert werden könnte; dass also nicht nur gelernt, sondern Gelerntes auch sofort umgesetzt werden könnte (Hopp u. Unger 2009, S. 81; vgl. auch Campbell 2008, S. 563). „Flexibel reagieren“ würde unter Bedingungen von „Nichtwissen“ dann kein Umsteuern der Wirkungen einer jeweiligen Peacebuilding-Maßnahme, sondern vielmehr ein erneutes Ausprobieren bedeuten. Dabei müsste weiterhin unter der Prämisse gearbeitet werden, dass selbst die Wirkungen von Maßnahmen, die nach bestem Wissen (um-)gestaltet werden, letztendlich nicht vorhersehbar sind.

Für praxisbezogene Forschung ergibt sich an dieser Stelle, zweitens, die Aufgabe, empirisch informiert und so detailliert wie möglich überhaupt den Status quo der „Peacebuilding-Routinen“ in internationalen Organisationen, (halb-)staatlichen Agenturen und NGOs zu beschreiben (vgl. etwa Schlichte u. Veit 2010). Die Arbeitsprozesse der Umsetzung von Peacebuilding sind bislang kaum empirisch 
erforscht worden. Ausgehend von Einsichten in den Status quo könnten dann organisations- und lerntheoretisch informierte Verbesserungs-Empfehlungen für einen Umgang mit „Nichtwissen“ formuliert werden, der im Idealfall Austauschprozesse zwischen unterschiedlichen Peacebuilding-Akteuren beinhalten würde (vgl. Hopp u. Unger 2009, S. 81-82). Spätestens an dieser Stelle wird auch deutlich, dass Fragen von „Koordination und Integration“ sich im Zuge einer Auseinandersetzung über den Umgang mit „Nichtwissen“ nicht von alleine erledigen. Dasselbe gilt auch für die Frage einer Beteiligung lokaler Akteure: Sollten lokale Akteure vor allem als Betroffene konzipiert werden - oder doch eher als Experten für ihre eigene soziale Realität? Wann im Analyseprozess würde es Sinn machen, vor allem lokale oder vor allem externe Mitarbeiter einzusetzen? Und wem sollte letzten Endes in welchen Fragen die Deutungshoheit zukommen?

Wenn aus dem Zustand des „Nichtwissens“ bis auf Weiteres nicht einfach ausgebrochen werden kann, dann stellt sich drittens die Frage, welchen Status hypothesenhaftes „Orientierungswissen“ (vgl. Friedrichs u. Kratochwil 2009, S. 706) haben soll bzw. wie damit umgegangen werden soll. Wenn die Erarbeitung von kausalem Steuerungswissen in komplexer sozialer Realität methodologisch mehr als problematisch ist, dann bestünde zumindest die Option, auf Basis von empirischer und theoretischer Plausibilität zu untersuchen, ob sich prozesshafte Zusammenhänge zwischen Peacebuilding-Maßnahmen und Aspekten sozialer Realität bzw. Formen von sozialem Wandel herstellen lassen, die mit dem übereinstimmen, was eine jeweilige Peacebuilding-Maßnahme als intendierte Wirkung hätte hervorbringen sollen - bzw. als nicht-intendierte Wirkung nicht hätte hervorbringen sollen (vgl. Leonhardt 2003, S. 61-63; Quack 2007, S. 39). Die Ergebnisse solcher Untersuchungen würden zwar kein Steuerungswissen liefern, wären aber zumindest dazu geeignet, Vorstellungen und Annahmen über die Wirkungen von Peacebuilding-Maßnahmen zu liefern, die hypothesenhafte Orientierung für die zukünftige Umsetzung ähnlicher Maßnahmen bieten könnten.

Solches Orientierungswissen wird in Form von „lessons learned“ bereits vielfach erarbeitet, allerdings meist auf sehr hohem Abstraktionsniveau und eher auf Ergebnisse denn auf Prozesse fokussiert. ${ }^{16}$ Bislang entsteht, wenn „lessons learned" präsentiert werden, meist der Eindruck, dass zwar vieles über die Wirkungen von Peacebuilding nicht gewusst wird, dass „lessons learned“ jedoch durchaus Steuerungswissen konstituieren. Eine solch kritische Einordnung wäre nur dann nicht notwendig, wenn, wie es in der Einleitung zu einem kürzlich erschienenen, soziologisch ausgerichteten Sammelband über „Interventionsgesellschaften“ heißt, „fast jeder Akteur auf der Systemebene“ bereits wüsste, dass eine Steuerung der Wirkungen von Peacebuilding nicht möglich ist (Daxner et al. 2010, S. 12); damit dürften sich dann sowohl der Wissenschafts- und Politikbetrieb als auch Praktikerinnen und Praktiker im Peacebuilding-System angesprochen füh-

16 Derzeit läuft zudem ein von der Deutschen Stiftung Friedensforschung gefördertes Forschungsprojekt zu der Frage, inwieweit „lessons learned“ innerhalb der UN absorbiert werden („organizational learning"), sodass einmal gewonnene Erkenntnisse für die zukünftige Umsetzung von Peacebuilding-Maßnahmen zur Verfügung stehen könnten (vgl. Benner et al. 2007). 
len. Davon, dass dies tatsächlich der Fall ist, kann aus unserer Sicht jedoch nicht ausgegangen werden (vgl. ebenso Campbell 2008, S. 563).

Wenn dem Stand der Forschung und Praxis im Bereich des Peacebuilding dann ein nur sehr mäßiges Zeugnis ausgestellt werden kann und für die Zukunft eine konstruktive wie reflektierte Auseinandersetzung mit „Nichtwissen“ empfohlen wird, dann ist damit keinesfalls gesagt, dass dies quasi automatisch zu einer Verbesserung von Peacebuilding - im Sinne einer Erhöhung der Chancen auf dauerhaften und selbsttragenden Frieden - beitragen muss. Zumal den Verbesserungspotenzialen durch Reformresistenzen aufseiten der Peacebuilding-Akteure (vgl. Lipson 2007; Campbell 2008) Grenzen gesetzt sind und es fraglich bleibt, in welchem Maß sich welcher Frieden überhaupt durch externe Interventionen fördern lässt (vgl. Manning 2004, S. 83-84; Barnett u. Zürcher 2009, S. 34). Doch wenn der Zweifel nicht ausgeräumt werden kann, ob sich potenziell „gute“ Intentionen überhaupt in friedenspolitisch „gute“ Taten übersetzen lassen, dann rührt dies nicht nur an der Art und Weise der Wissensproduktion innerhalb des Peacebuilding-Mainstreams, sondern auch an den Legitimierungspraktiken der vorherrschend „westlichen“ Interventionsansätze.

\section{Literatur}

Albrecht, Peter, und Paul Jackson. 2009. Security System Transformation in Sierra Leone, 1997-2007, Global Facilitation Network for Security Sector Reform. http://www.ssrnetwork.net/publications/security_s.php. Zugegriffen 29.05.2011.

Austin, Alex, Martina Fischer, und Oliver Wils (Hrsg.). 2003. Peace and Conflict Impact Assessment: Critical Views on Theory and Practice. Berghof Handbook Dialogue Series 1. Berlin: Berghof Zentrum für Konfliktforschung.

Baranyi, Stephen (Hrsg.). 2008a. The Paradoxes of Peacebuilding Post-9/11, Vancouver: University of British Columbia Press.

Baranyi, Stephen. 2008b. Introduction: What Kind of Peace is Possible in the Post-9/11 Era? In The Paradoxes of Peacebuilding Post-9/11, Hrsg. Stephen Baranyi, 3-31. Vancouver: University of British Columbia Press.

Baranyi, Stephen, und Kristiana Powell. 2008. Conclusion. In The Paradoxes of Peacebuilding Post-9/11, Hrsg. Stephen Baranyi, 293-316. Vancouver: University of British Columbia Press.

Barnett, Michael. 2006. Building a Republican Peace. Stabilizing States after War. International Security 30:87-112.

Barnett, Michael, und Christoph Zürcher. 2009. The Peacebuilder's Contract: How External Statebuilding Enforces Weak Statehood. In The Dilemmas of Statebuilding. Confronting the Contradictions of Postwar Peace Operations, Hrsg. Roland Paris und Timothy Sisk, 23-52. Abingdon/New York: Routledge.

Bell, Christine. 2009. Transitional Justice, Interdisciplinarity and the State of the 'Field' or 'Non-Field'. The International Journal of Transitional Justice 3:5-27.

Bendix, Daniel, und Ruth Stanley. 2008. Security Sector Reform in Africa: The Promise and the Practice of a New Donor Approach. ACCORD Occasional Paper Series Vol. 3 No. 2. Mount Edgecombe: African Centre for the Constructive Resolution of Disputes. 
Benner, Thorsten, Andrea Binder, und Philipp Rotmann. 2007. Learning to Build Peace? United Nations Peacebuilding and Organizational Learning: Developing a Research Framework. GPPi Research Paper 7. Berlin: Global Public Policy Institute.

Buckley-Zistel, Susanne. 2005. "The Truth Heals” ? Gacaca Jurisdictions and the Consolidation of Peace in Rwanda. Die Friedens-Warte 80:1-17.

Bush, Kenneth. 2003. PCIA Five Years On: The Commodification of an Idea. In Peace and Conflict Impact Assessment: Critical Views on Theory and Practice, Berghof Handbook Dialogue Series No. 1, Hrsg. Alex Austin, Martina Fischer und Oliver Wils, 3752. Berlin: Berghof Zentrum für Konfliktforschung.

Brounéus, Karen. 2010. The Trauma of Truth Telling: Effects of Witnessing in the Rwandan Gacaca Courts on Psychological Health. Journal of Conflict Resolution 54:408437.

Call, Charles T. (Hrsg.). 2007a. Constructing Justice And Security After War. Washington: United States Institute of Peace.

Call, Charles T. 2007b. Introduction: What We Know and Don't Know About Postconflict Justice and Security Reform. In Constructing Justice And Security After War, Hrsg. Charles T. Call, 3-28, Washington: United States Institute of Peace.

Call, Charles T., und Elizabeth M. Cousens. 2008. Ending Wars and Building Peace. International Responses to Warn-Torn Societies. International Studies Perspectives 9:1-21.

Campbell, Susanna P. 2008. (Dis)integration, Incoherence and Complexity in UN Postconflict Interventions. International Peacekeeping 15:556-569.

Campbell, Susanna P., und Anja T. Kaspersen. 2008. The UN's Reforms: Confronting Integration Barriers. International Peacekeeping, 15:470-485.

Caplan, Richard. 2007. From Collapsing States to Neo-Trusteeship: The Limits to Solving the Problem of 'Precarious Statehood' in the 21 ${ }^{\text {st }}$ Century. Third World Quarterly 2:231-244.

Chesterman, Simon. 2007. Ownership in Theory and in Practice: Transfer of Authority in UN Statebuilding Operations. Journal of Intervention and Statebuilding 1:3-26.

Chandler, David. 2000. Bosnia. Faking Democracy after Dayton. London/Sterling: Pluto Press.

Coulter, Chris, Mariam Persson, und Mats Utas. 2008. Young Female Fighters in African Wars. Conflict and Its Consequences. Uppsala: Nordiska Afrikainstitutet.

Daxner, Michael, Jan Free, Thorsten Bonacker, und Christoph Zürcher. 2010. Einleitung. In Interventionskultur. Zur Soziologie von Interventionsgesellschaften, Hrsg. Thorsten Bonacker, Jan Free, Michael Daxner und Christoph Zürcher, 7-18. Wiesbaden: VS Verlag für Sozialwissenschaften.

Dayton, Bruce W. 2009. Useful but Insufficient: Intermediaries in Peacebuilding. In Conflict Transformation And Peacebuilding. Moving From Violence To Sustainable Peace, Hrsg. Bruce W. Dayton und Louis Kriesberg, 61-73. Abingdon/New York: Routledge.

Dayton, Bruce W., und Louis Kriesberg. 2009 Introduction. In Conflict Transformation And Peacebuilding. Moving From Violence To Sustainable Peace, Hrsg. Bruce W. Dayton und Louis Kriesberg, 1-12. Abingdon/New York: Routledge.

De Zeeuw, Jeroen (Hrsg.). 2007. From Soldiers to Politicians. Transforming Rebel Movements After Civil War. Boulder/London: Lynne Rienner.

Doyle, Michael W., und Nicholas Sambanis. 2006. Making War and Building Peace. United Nations Peace Operations. Princeton/Woodstock: Princeton University Press. 
Duffield, Mark. 2002. Social Reconstruction and the Radicalization of Development: Aid as a Relation of Global Liberal Governance. Development and Change 33:1049-1071.

Duffield, Mark. 2005. Getting Savages to Fight Barbarians: Development, Security and the Colonial Present. Conflict, Security \& Development 5:142-159.

Duffield, Mark. 2008. Global Civil War: The Non-Insured, International Containment and Post-Interventionary Society. Journal of Refugee Studies 21:145-165.

Fortna, Virginia Page. 2008. Does Peacekeeping Work? Shaping Belligerents' Choices After Civil War. Princeton/Woodstock: Princeton University Press.

Fortna, Virginia Page, und Lise Morjé Howard. 2008. Pitfalls and Prospects in the Peacekeeping Literature. Annual Review of Political Science 11:283-301.

Free, Jan. 2010. Wege zu einer Soziologie moderner Friedenseinsätze. In Interventionskultur. Zur Soziologie von Interventionsgesellschaften, Hrsg. Thorsten Bonacker, Jan Free, Michael Daxner und Christoph Zürcher, 49-73. Wiesbaden: VS-Verlag für Sozialwissenschaften.

Friedrichs, Jörg, und Friedrich Kratochwil. 2009. On Acting and Knowing: How Pragmatism Can Advance International Relations Research and Methodology. International Organization, 63:701-31.

Gromes, Thorsten. 2003. Den Frieden abgewählt? Die Friedenskonsolidierung in Bosnien und Herzegowina und die Wahlen im Oktober 2002, HSFK-Report 4/2003. Frankfurt a. M.: Hessische Stiftung Friedens- und Konfliktforschung.

Hopp, Ulrike, und Barbara Unger. 2009. Time to Learn: Expanding Organisational Capacities in Conflict Settings. In Peacebuilding at the Crossroads. Dilemmas and Paths for Another Generation, Berghof Handbook Dialogue Series No. 7, Hrsg. Beatrix Schmelzle und Martina Fischer, 77-86. Berlin: Berghof Zentrum für Konfliktforschung.

Humphreys, Macartan, und Jeremy M. Weinstein. 2007. Demobilization and Reintegration. Journal of Conflict Resolution 51:531-567.

ICISS. 2001. The Responsibility To Protect. Report of the International Commission on Intervention and State Sovereignty. Ottawa: International Development Research Centre.

Jarstad, Anna K. 2008a. Power Sharing: Former Enemies in Joint Government. In From War to Democracy: Dilemmas of Peacebuilding, Hrsg. Anna K. Jarstad und Timothy D. Sisk, 105-133. Cambridge: Cambridge University Press.

Jarstad, Anna K. 2008b. Dilemmas of War-to-Democracy-Transitions: Theories and Concepts. In From War to Democracy: Dilemmas of Peacebuilding, Hrsg. Anna K. Jarstad und Timothy D. Sisk, 17-36. Cambridge: Cambridge University Press.

Jarstad, Anna K., und Timothy D. Sisk (Hrsg.). 2008. From War to Democracy: Dilemmas of Peacebuilding. Cambridge: Cambridge University Press.

Jennings, Kathleen M., und Anja T. Kaspersen. 2008. Introduction: Integration Revisited. International Peacekeeping 15:443-452.

Körppen, Daniela. 2007. Peace and Conflict Impact Assessment - Über die Utopie einer einheitlichen Methode. In Zivile Konfliktbearbeitung. Aktuelle Forschungsergebnisse, INEF-Report 85, Hrsg. Christoph Weller, 27-34. Duisburg: Institut für Entwicklung und Frieden.

Krasner, Stephen D. 2004. Governance Failures and Alternatives to Sovereignty. CDDRL Working Paper No.1. Stanford: Center on Democracy, Development, and The Rule of Law.

Lederach, John Paul. 2005. The Moral Imagination. The Art and Soul of Building Peace. New York/Oxford: Oxford University Press. 
Leonhardt, Manuela. 2003. Towards a Unified Methodology: Reframing PCIA. In Peace and Conflict Impact Assessment: Critical Views on Theory and Practice, Berghof Handbook Dialogue Series No. 1, Hrsg. Alex Austin, Martina Fischer und Oliver Wils, 53-66. Berlin: Berghof Zentrum für Konfliktforschung.

Lipson, Michael. 2007. Peacekeeping: Organized Hypocrisy? European Journal of International Relations 13:5-34.

Mac Ginty, Roger. 2006. No War, No Peace. The Rejuvenation of Stalled Peace Processes and Peace Accords. New York/Basingstoke: Palgrave Macmillan.

Mac Ginty, Roger. 2008. Indigenous Peace-Making Versus the Liberal Peace. Cooperation and Conflict 43:139-163.

Major, Claudia, Tobias Pietz, Elisabeth Schöndorf, und Wanda Hummel. 2011. Toolbox Krisenmanagement. Von der zivilen Krisenprävention bis zum Peacebuilding: Prinzipien, Akteure, Instrumente. Berlin: Stiftung Wissenschaft und Politik und Zentrum für Internationale Friedenseinsätze.

McKay, Susan, und Dyan Mazurana. 2004. Where are the Girls? Girls in Fighting Forces in Northern Uganda, Sierra Leone and Mozambique: Their Lives During and After War. Montréal: Rights \& Democracy.

Manning, Carrie. 2004. Elections and Political Change in Post-War Bosnia and Herzegowina. Democratization 11:60-86.

Mitchell, Christopher R. 2005. Conflict, Social Change and Conflict Resolution. An Enquiry, Berghof Handbook for Conflict Transformation. http://www.berghof-handbook. net/documents/publications/mitchell_handbook. Zugegriffen 29.05.2011.

Muggah, Robert (Hrsg.). 2009. Security and Post-Conflict Reconstruction. Dealing with Fighters in the Aftermath of War. Abingdon/New York: Routledge.

Narten, Jens. 2009. Dilemmas of Promoting "Local Ownership". The Case of Postwar Kosovo. In The Dilemmas Of Statebuilding. Confronting The Contradictions Of Postwar Peace Operations, Hrsg. Roland Paris und Timothy Sisk, 252-284. Abingdon/New York: Routledge.

Paris, Roland. 2004. At War's End: Building Peace after Civil Conflict. Cambridge: Cambridge University Press.

Paris, Roland. 2009. Understanding the "Coordination Problem" in Postwar Statebuilding. In The Dilemmas Of Statebuilding. Confronting The Contradictions Of Postwar Peace Operations, Hrsg. Roland Paris und Timothy Sisk, 53-78. Abingdon/New York: Routledge.

Paris, Roland. 2010. Saving Liberal Peacebuilding. Review of International Studies 36:337365.

Paris, Roland, und Timothy Sisk (Hrsg.). 2009. The Dilemmas Of Statebuilding. Confronting The Contradictions Of Postwar Peace Operations. Abingdon/New York: Routledge.

Paris, Roland, und Timothy Sisk. 2009a. Introduction: Understanding the Contradictions of Postwar Statebuilding. In The Dilemmas Of Statebuilding. Confronting The Contradictions Of Postwar Peace Operations, Hrsg. Roland Paris und Timothy Sisk, 1-20. Abingdon/New York: Routledge.

Paris, Roland, und Timothy Sisk. 2009b. Conclusion: Confronting the Contradictions. In The Dilemmas Of Statebuilding. Confronting The Contradictions Of Postwar Peace Operations, Hrsg. Roland Paris und Timothy Sisk, 304-315. Abingdon/New York: Routledge. 
Quack, Martin. 2007. Wirkungsanalysen in der zivilen Konfliktbearbeitung. In Zivile Konfliktbearbeitung. Aktuelle Forschungsergebnisse, INEF-Report 85, Hrsg. Christoph Weller, 35-44. Duisburg: Institut für Entwicklung und Frieden.

Reilly, Benjamin. 2008. Post-War Elections: Uncertain Turning Points of Transition. In From War to Democracy: Dilemmas of Peacebuilding, Hrsg. Anna K. Jarstad und Timothy D. Sisk, 157-181. Cambridge: Cambridge University Press.

Reich, Hannah. 2006. "Local Ownership" in Conflict Transformation Projects. Partnership, Participation or Patronage? Berghof Occasional Paper No. 27/2006. Berlin: Berghof Zentrum für Konfliktforschung.

Richmond, Oliver, und Jason Franks. 2007. Liberal Hubris? Virtual Peace in Cambodia. Security Dialogue 38:27-48.

Rubinstein, Robert A., Diana M. Keller, und Michael E. Scherger. 2008. Culture and Interoperability in Integrated Missions. International Peacekeeping 15:540-555.

Schlichte, Klaus, und Alex Veit. 2010. Drei Arenen. Warum Staatsbildung von außen so schwierig ist. In Interventionskultur. Zur Soziologie von Interventionsgesellschaften, Hrsg. Thorsten Bonacker, Jan Free, Michael Daxner und Christoph Zürcher, 261-268. Wiesbaden: VS Verlag für Sozialwissenschaften.

Schmelzle, Beatrix, und Martina Fischer (Hrsg.). 2009. Peacebuilding at the Crossroads. Dilemmas and Paths for Another Generation, Berghof Handbook Dialogue Series No.

7. Berlin: Berghof Zentrum für Konfliktforschung.

Smith, Dan. 2004. Towards a Strategic Framework for Peacebuilding: Getting Their Act Together. Overview report of the Joint Utstein Study of Peacebuilding. Oslo: Royal Norwegian Ministry of Foreign Affairs.

Shaw, Rosalind. 2007. Memory Frictions: Localizing the Truth and Reconciliation Commission in Sierra Leone. International Journal of Transitional Justice 1:183-207.

Stengel, Frank A., und Christoph Weller. 2010. Action Plan or Faction Plan? Germany's Eclectic Approach to Conflict Prevention. International Peacekeeping 17:93-107.

Stromseth, Jane, David Wippman, und Rosa Brooks. 2006. Can Might Make Rights? Building the Rule of Law After Military Interventions. Cambridge: Cambridge University Press.

UN Secretary General. 1992. An Agenda for Peace. Preventive Diplomacy, Peacemaking and Peace-Keeping. http://www.un.org/Docs/SG/agpeace.html. Zugegriffen 27.6.2011.

\section{Autorenangaben:}

Prof. Dr. Sven Chojnacki,

Freie Universität Berlin, Otto-Suhr-Institut für Politikwissenschaft, Ihnestr. 26,

14195 Berlin, svencho@zedat.fu-berlin.de

[Korrespondenzautor]

Anne Menzel, Freie Universität Berlin, Otto-Suhr-Institut für Politikwissenschaft, Ihnestr. 26, 14195 Berlin, amenzel@zedat.fu-berlin.de 